Monatsh Math 153, 309-346 (2008)

DOI 10.1007/s00605-007-0518-9

Printed in The Netherlands

\title{
Shift coordinates, stretch lines and polyhedral structures for Teichmüller space
}

\author{
By \\ Athanase Papadopoulos ${ }^{1,2}$ and Guillaume Théret $^{1,3}$ \\ ${ }^{1}$ Université Louis Pasteur and CNRS, Strasbourg, France \\ ${ }^{2}$ Max-Planck-Institut für Mathematik, Bonn, Germany \\ ${ }^{3}$ University of Aarhus, Denmark
}

Received January 15, 2007; accepted in final form September 18, 2007

Published online February 8, 2008 (C) Springer-Verlag 2008

\begin{abstract}
This paper has two parts. In the first part, we study shift coordinates on a sphere $S$ equipped with three distinguished points and a triangulation whose vertices are the distinguished points. These coordinates parametrize a space $\mathscr{T}(S)$ that we call an unfolded Teichmüller space. This space contains Teichmüller spaces of the sphere with $\mathfrak{b}$ boundary components and $\mathfrak{p}$ cusps (which we call generalized pairs of pants), for all possible values of $\mathfrak{b}$ and $\mathfrak{p}$ satisfying $\mathfrak{b}+\mathfrak{p}=3$. The parametrization of $\widetilde{T}(S)$ by shift coordinates equips this space with a natural polyhedral structure, which we describe more precisely as a cone over an octahedron in $\mathbb{R}^{3}$. Each cone over a simplex of this octahedron is interpreted as a Teichmüller space of the sphere with $\mathfrak{b}$ boundary components and $\mathfrak{p}$ cusps, for fixed $\mathfrak{b}$ and $\mathfrak{p}$, the sphere being furthermore equipped with an orientation on each boundary component. There is a natural linear action of a finite group on $\mathscr{T}(S)$ whose quotient is an augmented Teichmüller space in the usual sense. We describe several aspects of the geometry of the space $\mathscr{T}(S)$. Stretch lines and earthquakes can be defined on this space. In the second part of the paper, we use the shift coordinates to obtain estimates on the behaviour of stretch lines in the Teichmüller space of a surface obtained by gluing hyperbolic pairs of pants. We also use the shift coordinates to give formulae that express stretch lines in terms of Fenchel-Nielsen coordinates. We deduce the disjointness of some stretch lines in Teichmüller space. We study in more detail the case of a closed surface of genus 2 .
\end{abstract}

\section{Mathematics Subject Classification: 32G15, 30F60, 57N16, 53A35, 30F45}

Key words: Ideal triangulation, hyperbolic structure, horocyclic foliation, measured foliation, stretch line, shift parameters, Fenchel-Nielsen parameters, earthquake, Teichmüller space, unfolded Teichmüller space

\section{Introduction}

We first establish some notations. Let $\mathfrak{g}, \mathfrak{b}$ and $\mathfrak{p}$ be nonnegative integers and let $S_{\mathfrak{g}, \mathfrak{b}, \mathfrak{p}}$ be an oriented connected surface of genus $\mathfrak{g}$ with $\mathfrak{b}$ boundary components and $\mathfrak{p}$ punctures. We shall always assume that $S_{\mathfrak{g}, \mathfrak{b}, \mathfrak{p}}$ has negative Euler characteristic. A hyperbolic structure (or hyperbolic metric) on $S_{\mathfrak{g}, \mathfrak{b}, \mathfrak{p}}$ is a complete Riemannian metric of constant curvature -1 which has finite area and such that each boundary component of $S_{\mathfrak{q}, \mathfrak{b}, \mathfrak{p}}$ is a closed geodesic. The Teichmüller space $\mathscr{T}_{\mathfrak{g}, \mathfrak{b}, \mathfrak{p}}=\mathscr{T}\left(S_{\mathfrak{g}, \mathfrak{b}, \mathfrak{p}}\right)$ of $S_{\mathfrak{g}, \mathfrak{b}, \mathfrak{p}}$ is the space of isotopy classes of hyperbolic metrics on $S_{\mathfrak{g}, \mathfrak{b}, \mathfrak{p}}$. It is equipped with a natural topology which makes it homeomorphic to $\mathbb{R}^{6 \mathfrak{g}-6+3 \mathfrak{b}+2 \mathfrak{p}}$, see e.g. [6] and [12].

We shall usually denote the closed surface of genus $\mathfrak{g}$ by $S_{\mathfrak{g}}$ instead of $S_{\mathfrak{g}, 0,0}$. 
Let $P=\left\{p_{1}, \ldots, p_{n}\right\} \subset S_{\mathfrak{g}}$ be a finite collection of points, which we call the distinguished points. We assume that the Euler characteristic of the punctured surface $S_{\mathfrak{g}} \backslash P$ is negative. By a hyperbolic structure on the pair $\left(S_{\mathfrak{g}}, P\right)$, we mean a complete finite area hyperbolic structure on a surface $S_{\mathfrak{g}, \mathfrak{b}, \mathfrak{p}}$ obtained from $S_{\mathfrak{g}}$ by deleting $\mathfrak{p}$ points among the distinguished points $(0 \leqslant \mathfrak{p} \leqslant n)$ and making them punctures for the hyperbolic structure, and deleting an open disk around each of the remaining $\mathfrak{b}=n-\mathfrak{p}$ distinguished points, and making the surface around each of these $\mathfrak{b}$ points a surface with boundary.

Finally, a signed hyperbolic structure on the pair $\left(S_{\mathfrak{g}}, P\right)$ is a hyperbolic structure together with an orientation on each boundary component of the resulting hyperbolic surface. The collection of isotopy classes of signed hyperbolic structures on $\left(S_{\mathrm{q}}, P\right)$ forms a space which we call the unfolded Teichmüller space of $\left(S_{\mathfrak{g}}, P\right)$, and which we denote by $\widetilde{T}\left(S_{\mathfrak{g}}, P\right)$ (and sometimes $\mathscr{T}\left(S_{\mathfrak{g}}\right)$, if the set of distinguished points is understood). The unfolded Teichmüller space $\widetilde{T}\left(S_{\mathfrak{g}}, P\right)$ is the union of a finite collection of Teichmüller spaces $\mathscr{T}\left(S_{\mathfrak{g}, \mathfrak{b}, \mathfrak{p}}\right)$ of surfaces $S_{\mathfrak{g}, \mathfrak{b}, \mathfrak{p}}$ obtained from $S_{\mathfrak{g}}$ by making each of the distinguished points either a puncture or a boundary component. We shall give a parametrization of the unfolded Teichmüller space of $\left(S_{\mathfrak{g}}, P\right)$ by using shift coordinates on the edges of an ideal triangulation of the pair $\left(S_{\mathrm{g}}, P\right)$, that is, a triangulation of $S$ whose vertex set is $P$.

The parameterization of hyperbolic structures on surfaces using shift coordinates on edges of ideal triangulations was introduced by Thurston, who used these parameters in relation to stretch maps between hyperbolic surfaces and in his study of the asymmetric metric he defined on Teichmüller space, see [11]. Shift coordinates can also be used in the description of earthquakes, see [11]. Shift coordinates were used in [9], together with the analogously defined shift parameters for measured foliations, in the study of the extension of the earthquake flow to Thurston's boundary of Teichmüller space. Shift coordinates were also used in [10] as coordinates for homeomorphisms of the circle. Finally, shift coordinates were used by several authors in the quantization theory of Teichmüller space (see $[1]-[5])$.

The parametrization of $\widetilde{T}\left(S_{\mathfrak{g}}, P\right)$ by shift coordinates on the edges of an ideal triangulation can be regarded as a unifying way of parametrizing the various copies of the Teichmüller spaces of the surfaces $S_{\mathfrak{q}, \mathfrak{b}, \mathfrak{p}}$ with $\mathfrak{b}+\mathfrak{p}=n$. We shall see that this parametrization endows this union with a natural structure of a polyhedron. In particular, there is a natural topology on the unfolded Teichmüller space, which makes it a connected space, more precisely, a space homeomorphic to $\mathbb{R}^{6 \mathfrak{g}-6+3 \mathfrak{n}}$. For the general values of $\mathfrak{b}$ and $\mathfrak{p}$, each Teichmüller space $\mathscr{T}\left(S_{\mathfrak{g}, \mathfrak{b}, \mathfrak{p}}\right)$ is represented by several copies inside the space $\widetilde{T}\left(S_{\mathfrak{g}}, P\right)$, the elements of these individual Teichmüller spaces being obtained from elements of $\widetilde{T}\left(S_{\mathfrak{g}}, P\right)$ by forgetting signs.

We shall study in detail the case where $S_{\mathfrak{g}}$ is a sphere $S=S^{2}$, with $\operatorname{Card}(P)=3$. In this case, we shall describe in detail the polyhedral structure for $\mathscr{T}\left(S_{\mathfrak{g}}, P\right)$ (which at the same time defines the topology on that space). The polyhedron parametrizing $\mathscr{T}\left(S_{\mathfrak{g}}, P\right)$ is the space $\mathbb{R}^{3}$ seen as the cone from the origin over an octahedron that contains the origin in its interior. In this description, the various Teichmüller spaces $\mathscr{T}\left(S_{\mathfrak{g}, \mathfrak{b}, \mathfrak{p}}\right)$ (with $\mathfrak{g}=0$ and $\mathfrak{b}+\mathfrak{p}=3$ ) referred to 
above are the cones over the open faces of this polyhedron. We shall discuss the dependence of this polyhedral structure on the ideal triangulation.

We note that Feng Luo also studied a parametrization of Teichmüller space by a convex polytope in the recent paper [8].

It is a recurrent theme in low-dimensional topology that geometric structures on (generalized) pairs of pants are building blocks for geometric structures on general surfaces. This theme will appear in the second part of this paper.

We recall that if $\mu$ is a complete geodesic lamination on a surface $S$, if $h$ is a hyperbolic structure on $S$ and if $t$ is a real number, then an $e^{t}$-stretch map directed by $\mu$ is a map from $S$ to itself which is isotopic to the identity on $S$, for which $\mu$ is a maximally stretched lamination and which transforms $h$ into another hyperbolic structure $h^{t}$ whose distance from $h$ (with respect to Thurston's asymmetric metric) is equal to $t$, if $t$ is positive. (We note by the way that if $t$ is negative, the distance in general is not equal to $-t$.) In the shift coordinates associated to $\mu$, the transformation consists in multiplying each coordinate by the factor $e^{t}$. The map $t \mapsto h^{t}$ is called the stretch line starting at $h=h^{0}$ and directed by $\mu$. This line is a geodesic for Thurston's asymmetric metric.

Stretch lines and earthquake lines have a nice description in the polyhedral structure of the unfolded Teichmüller space $\widetilde{T}\left(S_{\mathfrak{g}}, P\right)$. These lines follow linear segments with respect to this polyhedral structure.

In this paper, we study the particular case where the lamination $\mu$ is an ideal triangulation on a surface obtained by gluing pairs of pants. We establish explicit formulae that give relations between the Fenchel-Nielsen coordinates of the Teichmüller spaces of $S_{\mathfrak{g}, \mathfrak{b}, \mathfrak{p}}$ and the shift coordinates. In particular, we give an expression of stretch lines in terms of Fenchel-Nielsen coordinates. We use these formulae for the study of the behaviour of stretch lines. In particular, we can see the disjointness of certain stretch lines in Teichmüller space.

We would like to thank an anonymous referee for his (or her) careful reading of the original manuscript.

\section{A review of ideal triangulations and shift coordinates}

2.1. Triangulation. Consider a closed surface with distinguished points $\left(S_{\mathfrak{g}}, P\right)$. A triangulation of this pair is a cell decomposition of $S_{\mathrm{g}}$ into triangles whose set of vertices is the set $P$. Note that we do not require that the triangulation be a realization of a simplicial complex (that is, the two endpoints of an edge may be equal, and so on).

Given a triangulation $\mu$ of $\left(S_{\mathfrak{g}}, P\right)$ and a vertex of this triangulation, we shall sometimes talk about a half-edge of $\mu$ (instead of an edge of $\mu$ ) abutting on that vertex, in order to record one (or possibly both) of the ends of a given edge that abut on that vertex.

2.2. Ideal triangulation. We recall that a hyperbolic ideal triangle is a surface with boundary which is isometric to the convex hull in the hyperbolic plane $\mathbb{U}^{2}$ of three distinct points in the boundary of that space. The center of an ideal triangle is the intersection point of the three perpendiculars drawn from each ideal vertex to the edge opposite to it. 
Consider a surface $S_{\mathfrak{g}, \mathfrak{b}, \mathfrak{p}}$ equipped with a hyperbolic metric. An ideal triangulation $\mu$ of $S_{\mathfrak{g}, \mathfrak{b}, \mathfrak{p}}$ is a decomposition of $S_{\mathfrak{g}, \mathfrak{b}, \mathfrak{p}}$ by disjoint geodesic lines, called the edges of $\mu$, satisfying the following:

- each end of an edge either converges to a cusp or spirals around a closed geodesic which could be either an interior geodesic or a boundary geodesic;

- the completion of each component of the surface $S_{\mathfrak{g}, \mathfrak{b}, \mathfrak{p}}$ cut along the edges of $\mu$ is isometric to a hyperbolic ideal triangle.

It will often be useful to consider the hyperbolic surface $S_{\mathfrak{g}, \mathfrak{b}, \mathfrak{p}}$ equipped with its ideal triangulation as a surface obtained by assembling a collection of hyperbolic ideal triangles using isometries between their boundary edges. In this case, the decomposition of $S_{\mathfrak{g}, \mathfrak{b}, \mathfrak{p}}$ by the ideal triangulation is a record of the edges of the ideal triangles that we started with, together with the gluing maps between them.

Let $\left(S_{\mathrm{g}}, P\right)$ be a closed surface with distinguished points, let $h$ denote a signed hyperbolic structure on the pair $\left(S_{\mathfrak{g}}, P\right)$ and let $\mu$ be a triangulation of the pair $\left(S_{\mathfrak{g}}, P\right)$. Let $S_{\mathfrak{g}, \mathfrak{b}, \mathfrak{p}}$ be the type of the corresponding hyperbolic surface. Then, there is a canonical ideal triangulation of $S_{\mathfrak{g}, \mathfrak{b}, \mathfrak{p}}$ associated to $\mu$ (and we shall also denote by $\mu$ the ideal triangulation), which is characterized by the following properties:

- if a distinguished point in $P$, after equipping the surface $\left(S_{\mathfrak{g}}, P\right)$ with the signed hyperbolic structure $h$, becomes a cusp for this hyperbolic structure, then the half-edges of $\mu$ that have this distinguished point as vertex converge to the corresponding cusp;

- if a distinguished point in $P$, after equipping the surface $\left(S_{\mathfrak{g}}, P\right)$ with the signed hyperbolic structure $h$, becomes a boundary component for this hyperbolic structure, then the half-edges of $\mu$ that have this distinguished point as vertex spiral around the boundary geodesic, in the direction indicated by the orientation of the boundary geodesic induced by the signed hyperbolic structure $h$.

2.3. Flip. Consider again a closed surface with distinguished points $\left(S_{\mathfrak{g}}, P\right)$ equipped with a triangulation $\mu$. Let $e$ be an edge of $\mu$ that is on the boundary of two distinct triangles $T_{1}$ and $T_{2}$. A flip on $\left(S_{\mathfrak{g}}, \mu\right)$ associated to the edge $e$ is a move that transforms the triangulation $\mu$ into another triangulation as follows. Let $Q$ be the quadrilateral defined as the union $T_{1} \cup T_{2}$ glued along the edge $e$. Note that the interior of $Q$ is embedded in $S_{\mathfrak{g}}$. The flip operation consists in deleting the edge $e$ from the set of edges of $\mu$ and replacing it by an arc which represents the other diagonal of $Q$. Thus, the flip operation replaces the triangulation $\mu$ by a new triangulation. Note that if the two sides of some edge of a triangulation of $S_{\mathfrak{g}}$ are equal, then no flip can be performed on that edge.

When the surface is equipped with a hyperbolic structure, a flip operation on $\mu$ gives naturally rise to a flip operation on the corresponding ideal triangulation. Such a flip operation on an ideal triangulation consists in replacing an ideal quadrilateral equipped with a diagonal by the same ideal quadrilateral equipped with its other diagonal.

2.4. Shift coordinates for hyperbolic structures. Consider a hyperbolic surface $S_{\mathfrak{g}, \mathfrak{b}, \mathfrak{p}}$ equipped with an ideal triangulation $\mu$. Each edge $e$ of $\mu$ has two 

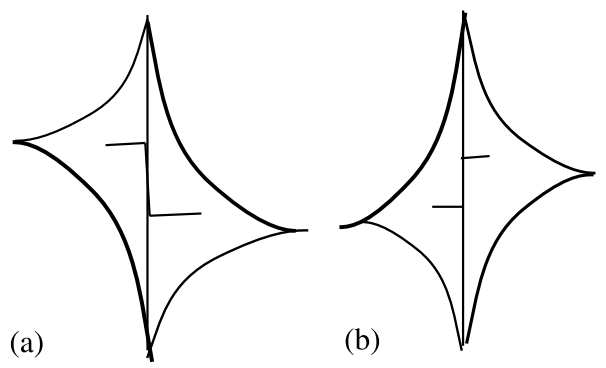

Figure 1. Shift coordinates for hyperbolic structures. Case (a) corresponds to a negative shift and case (b) corresponds to a positive shift

distinguished points, which are the orthogonal projections on $e$ of the centers of the two ideal triangles that are adjacent to it. (Note that these two triangles may be equal.) We define a signed distance between the two distinguished points in the following way. The absolute value of this quantity is the distance (measured on the edge $e$ ) between these points, measured using the hyperbolic metric, and the sign is determined by the sense of the shearing along the edge $e$ that can be performed to carry one of these points to the other one. The sign is taken to be positive (respectively negative) if the shearing is to the left (respectively to the right) (see Figure 1). Note that the orientation on the surface suffices to give a well-defined notion of left and right shear along every edge of $\mu$. The signed distance between the two distinguished points on $e$ is the shift parameter on that edge, with respect to the hyperbolic structure considered.

The collection of shift parameters on the various edges of $\mu$ completely determines the gluing between the ideal triangles, and therefore, it completely determines the isometry type of the hyperbolic surface $S$. Isotopic hyperbolic structures have the same signed shift parameters, and therefore the shift parameters can also be used as parameters for isotopy classes of hyperbolic structures on the surface, that is, for elements of the Teichmüller space of $S$.

One of the features of the shift parameters which can make them more advantageous than the Fenchel-Nielsen parameters is that shift parameters can be used as parameters for signed hyperbolic structures on the surface with distinguished points $\left(S_{\mathfrak{g}}, P\right)$. Indeed, we shall see that the shift parameters can be defined by assigning real numbers to the edges of the original triangulation $\mu$ on $S_{\mathfrak{g}}$ such that these real numbers determine a hyperbolic structure on the pair $\left(S_{\mathrm{g}}, P\right)$ together with, for each edge of $\mu$, an information on whether the end of this edge (with is geodesic for this metric) converges to a puncture or spirals around a boundary closed geodesic of the hyperbolic structure considered. Furthermore, for each end of a bi-infinite geodesic representing an edge of $\mu$ that spirals around a boundary closed geodesic, the shift parameters on $\mu$ determine the sense of spiraling. This sense of spiraling determines an orientation on each boundary component of the hyperbolic surface.

2.5. Shift coordinates for measured foliations transverse to $\boldsymbol{\mu}$. We consider measured foliations in the sense of Thurston, as described e.g. in [6] and [11]. Let $F$ 

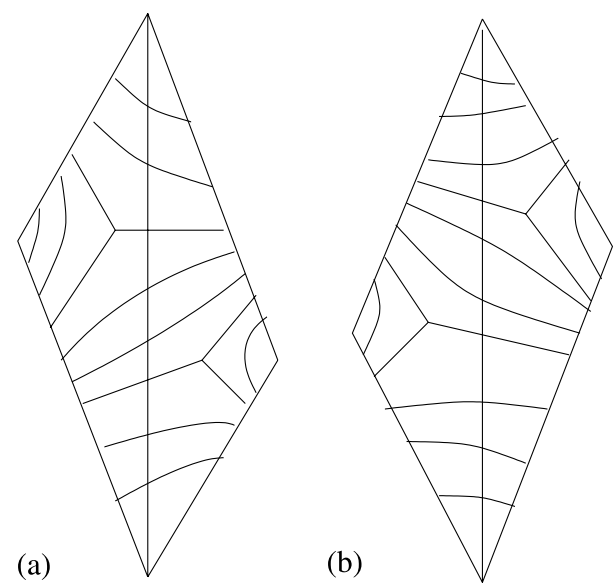

Figure 2. Shift coordinates for measured foliations. Case (a) corresponds to a negative shift and case (b) corresponds to a positive shift

be a measured foliation on $S_{\mathfrak{g}, \mathfrak{b}, \mathfrak{p}}$ which is transverse to $\mu$. Since each component of $S_{\mathfrak{g}, \mathfrak{b}, \mathfrak{p}} \backslash \mu$ is a triangle, it follows from an Euler characteristic argument that all the singularities of $F$ are three-pronged and that each component of $S_{\mathfrak{g}, \mathfrak{b}, \mathfrak{p}} \backslash \mu$ contains exactly one singularity. In this way, the measured foliation $F$ induces on each edge $e$ of $\mu$ two distinguished points, which are the feet of the singular leaves of $F$ that abut on that edge from its two sides. A signed transverse measure between these two points is then defined in the following way. The absolute value of this quantity is the transverse measure of the segment of $e$ bounded by these two distinguished points, and the sign is determined by the sense of shearing defined by the two singular leaves hitting these points. The convention for the sign is analogous to the one for the sign defining the shift parameters for hyperbolic structures that we discussed in Sect. 2.4 above (see Figure 2). This signed transverse measure is the shift parameter induced by the measured foliation on the edge $e$ of $\mu$. As in the case of hyperbolic structures, the set of shift parameters on the various edges of $\mu$ completely determines the gluing between the foliations restricted to the components of $S \backslash \mu$, and therefore, it completely determines the isotopy class of the foliation $F$.

2.6. Generalized pairs of pants and their canonical involutions. A generalized hyperbolic pair of pants is a hyperbolic surface homeomorphic to a sphere with $\mathfrak{b}$ boundary components and $\mathfrak{p}$ cusps, with $\mathfrak{b}+\mathfrak{p}=3$. We now describe the decomposition of a generalized pair of pants into two isometric generalized right-angled hexagons.

Consider a right-angled hexagon in the hyperbolic space $\mathbb{U}^{2}$. We can make the length of one of its edges tend to zero while keeping fixed the two edges that form with the degenerating edge a triple of edges which are pairwise non-adjacent, and keeping all the angles to be right angles. Then, the vertices of the degenerating edge converge to a single point on the boundary of $\mathbb{t}^{2}$, and the limit of the rightangled hexagon is called a degenerate right-angled hexagon with one edge at infinity (see Figure 3 for a picture of such a hexagon). Likewise, we can make 

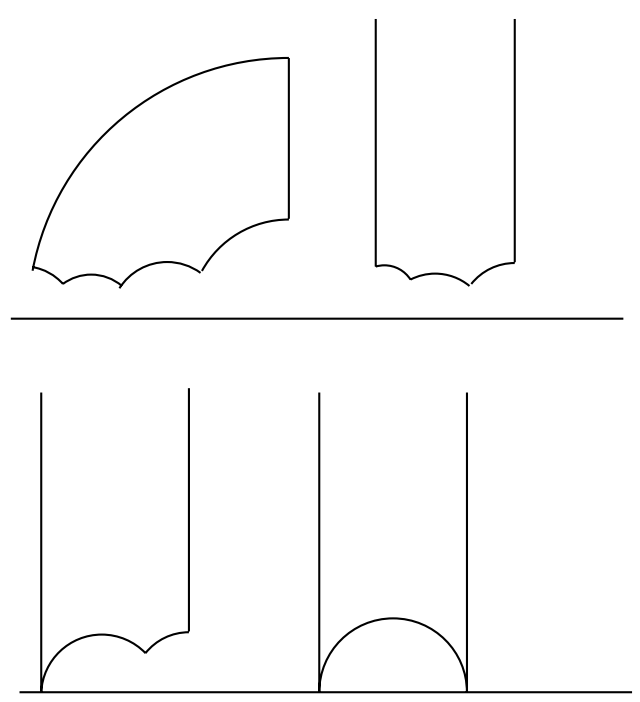

Figure 3. These four generalized polygons represent, respectively a right-angled hexagon and three degenerate right-angled hexagons in the upper half-plane model of the hyperbolic plane

the lengths of two edges of a right-angled hexagon which are separated by an edge tend to zero, keeping fixed the length of the edge that makes with this pair of edges a triple of pairwise non-adjacent edges and keeping the remaining angles to be right angles. Finally, we can make three pairwise non-adjacent edges of the rightangled hexagon tend to zero. We obtain in this manner degenerate right-angled hexagons with one, two or three edges at infinity. Note that a degenerate rightangled hexagon with three edges at infinity is a hyperbolic ideal triangle. A generalized right angled hexagon is either a genuine or a degenerate right-angled hexagon. This definition of a generalized right-angled hexagon as a limit of a sequence of (genuine) right-angled hexagons is useful for studying degenerations of hyperbolic surfaces. With such a definition, the trigonometric formulae giving the lengths of the edges of the generalized right-angled hexagons are obtained as limits of corresponding formulae for right-angled hexagons. From the formulae for distances in right-angled hexagons (see [6] or [7]), it is easy to see that a degenerate right-angled hexagon with one edge (respectively two, or three edges) at infinity is determined up to isometry by the lengths of the edges that make with the edge (respectively, the edges) at infinity a triple of edges which are pairwise non-adjacent (the length of an edge at infinity being, by definition, equal to zero). The decomposition of a generalized hyperbolic pair of pants into two isometric generalized right-angled hexagons is obtained by taking the union of the three geodesics that join perpendicularly pairs of boundary closed geodesics, in the case there are three boundary geodesics. In the other cases, we take the union of the geodesics that join a boundary closed geodesic to a cusp or that join a cusp to a cusp. We call such a geodesic a seam of the generalized pair of pants. From this decomposition of a generalized pair of pants into two generalized right-angled hexagons, it is easy to see that any hyperbolic structure on a generalized pair of 
pants is completely determined by the lengths of its boundary geodesics, a cusp being considered as a boundary geodesic of length zero. In this sense, the hyperbolic structure is rigid at a cusp. For instance, a degenerate right-angled hexagon with three edges at infinity is a hyperbolic ideal triangle which, as is well-known, is unique up to isometry. In the same way, any two degenerate hyperbolic pairs of pants that are spheres with three cusps are isometric.

We shall use the fact that any generalized hyperbolic pair of pants $P$ has a canonical involution $\iota$, which is the unique isometry that fixes the seams and that interchanges the two generalized right-angled hexagons that compose it.

\section{Horocyclic foliations and completeness}

3.1. Horocyclic foliations. A hyperbolic ideal triangle is equipped with a natural foliation, called its horocyclic foliation. This is a partial foliation (that is, a foliation of a subsurface of the ideal triangle) whose leaves are pieces of horocycles of $\mathbb{M}^{2}$ that are centered at the three ideal vertices, the nonfoliated region being a small triangle bounded by three pieces of horocycles that meet tangentially at their boundary points, as represented in Figure 4.

Let $g$ be a hyperbolic structure on a surface $S_{\mathfrak{g}, \mathfrak{b}, \mathfrak{p}}$ and let $\mu$ be an ideal triangulation on that surface. There is a natural measured foliation $F_{\mu}(g)$ which is associated to the pair $(g, \mu)$, and which is called the horocyclic measured foliation. It is obtained by assembling the horocyclic foliations of the components of $S_{\mathfrak{g}, \mathfrak{b}, \mathfrak{p}} \backslash \mu$, and it has a natural transverse measure which is determined by the fact that on the edges of $\mu$, the measure coincides with hyperbolic length (see [11] Sect. 2).

3.2. Holonomy type. Let $S_{\mathfrak{g}, \mathfrak{b}, \mathfrak{p}}$ be a surface equipped with a hyperbolic structure. There is a developing map from the metric universal covering space $\tilde{S}_{\mathfrak{g}, \mathfrak{b}, \mathfrak{p}}$ of $S_{\mathfrak{g}, \mathfrak{b}, \mathfrak{p}}$ into the hyperbolic plane $\mathbb{U}^{2}$. Recall that this map is a local isometry that arises as one tries to extend a coordinate chart of the hyperbolic structure to a "global chart", by gluing the charts that one encounters following paths along the surface $S_{\mathfrak{q}, \mathfrak{b}, \mathfrak{p}}$. The developing map is well-defined up to composition by an isometry of $\mathbb{\boxplus}^{2}$ which conjugates the action of the fundamental group $\pi_{1}\left(S_{\mathfrak{g}, \mathfrak{b}, \mathfrak{p}}\right)$ on $\tilde{S}_{\mathfrak{g}, \mathfrak{b}, \mathfrak{p}}$ to an action of $\pi_{1}\left(S_{\mathfrak{g}, \mathfrak{b}, \mathfrak{p}}\right)$ on $\mathbb{H}^{2}$ by isometries. There is an associated representation of $\pi_{1}\left(S_{\mathfrak{g}, \mathfrak{b}, \mathfrak{p}}\right)$

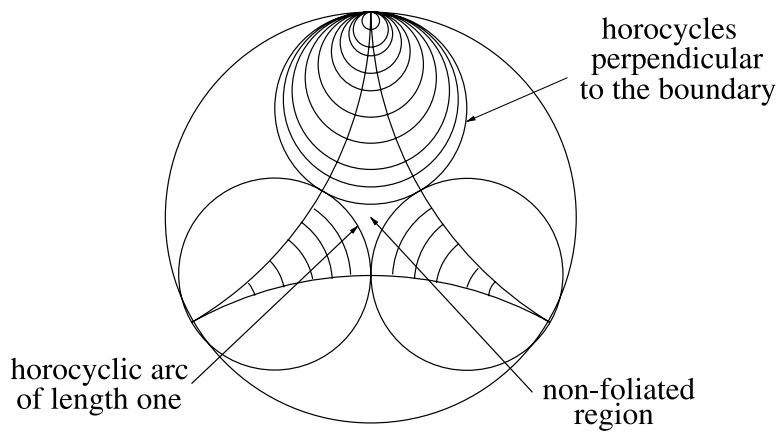

Figure 4. The horocyclic foliation of an ideal triangle 
into the group $\operatorname{Isom}^{+}\left(\mathbb{Q}^{2}\right)$ of orientation-preserving isometries of $\mathbb{M}^{2}$, called the holonomy representation, such that the developing map is equivariant with respect to the action of $\pi_{1}\left(S_{\mathfrak{g}, \mathfrak{b}, \mathfrak{p}}\right)$ on $\tilde{S}_{\mathfrak{g}, \mathfrak{b}, \mathfrak{p}}$ and the action on $\mathbb{Q}^{2}$ of the image of the holonomy representation (see Thurston [12] Sect. 3.4). With respect to this representation, the holonomy of a closed curve representing an element of $\pi_{1}\left(S_{\mathfrak{q}, \mathfrak{b}, \mathfrak{p}}\right)$ is the isometry of $\mathbb{Q}^{2}$ that is associated by the holonomy representation. We shall talk below about the holonomy type of a closed curve on $S_{\mathfrak{q}, \mathfrak{b}, \mathfrak{p}}$, and this needs some explanation. The notion of holonomy refers to a representation of the fundamental group of the surface. Although the holonomy of a closed curve in $S_{\mathfrak{g}, \mathfrak{b}, \mathfrak{p}}$, as an element of Isom ${ }^{+}\left(\mathbb{Q}^{2}\right)$ is not well-defined (it depends on the choice of an orientation for that closed curve, on the choice of a basepoint for the fundamental group and so on), the type of the holonomy (that is, whether it is parabolic or hyperbolic) is independent of any choice. Thus, we can talk about the holonomy type of a closed curve on $S_{\mathfrak{g}, \mathfrak{b}, \mathfrak{p}}$.

Let $S_{\mathfrak{g}}$ be a closed surface with a set $P$ of distinguished points equipped with a hyperbolic structure. For each distinguished point, we can take a simple closed curve that is homotopic to that point, and consider its holonomy type. We shall say that the distinguished point is of parabolic type (respectively hyperbolic type) if the holonomy of such a closed curve is parabolic (respectively hyperbolic). If the distinguished point is of parabolic type, then we shall say that the length of the distinguished point is zero. If the distinguished point is of hyperbolic type, then we shall say that the length of this distinguished point is the length of the boundary geodesic to which it is homotopic. It follows from the construction of the holonomy representation that in the case of hyperbolic holonomy, the length of a distinguished point is equal to the translation distance of any element of $\operatorname{Isom}^{+}\left(\mathbb{H}^{2}\right)$ representing the holonomy of a closed curve that is homotopic to that distinguished point.

3.3. Completeness. Let us consider a hyperbolic surface $\left(S_{\mathfrak{p}}, P\right)$ equipped with an ideal triangulation $\mu$ obtained by gluing a collection of hyperbolic ideal triangles by isometries between their boundary edges. The completeness of this hyperbolic surface depends on the geometric type of the neighborhoods of the punctures (that is, the vertices of the triangulation). There is a detailed discussion of completeness for such metrics in Thurston [12] Sect. 3.4. To understand the geometric type of $S_{\mathfrak{g}, \mathfrak{b}, \mathfrak{p}}$ near a puncture, it is useful to study the behaviour at that puncture of the horocyclic foliation associated to the ideal triangulation $\mu$. In the next proposition, we summarize a few facts that will be useful to us.

Proposition 3.1. Let $\left(S_{\mathfrak{g}}, P\right)$ be a surface with distinguished points, equipped with an ideal triangulation $\mu$, and consider a finite area hyperbolic structure $h$ on the punctured surface $S_{\mathfrak{g}} \backslash P$. Then, any puncture a of $S_{\mathfrak{g}} \backslash P$ is of one of the following two types:

(1) the puncture a has an annular neighborhood on which $F$ induces a foliation by closed leaves that are centered at that puncture;

(2) the puncture a has an annular neighborhood equipped with a foliation induced by $F$ which has no closed leaves. 
In Case (1), the puncture is a cusp, and we can choose a neighborhood $N$ of the puncture, equipped with the foliation induced by $F$, in such a way that $N$ is isometric to the quotient of a subset $N^{\prime}=\left\{z=x+i y \mid y>y_{0}\right\}$ of $\mathbb{U}^{2}$ (for some $\left.y_{0}>0\right)$ by the action of the map $z \mapsto z+1$. The foliation on $N$ is the quotient of the foliation of $N^{\prime}$ by horocycles centered at the point $\infty$. Furthermore, the closure in $S_{\mathfrak{g}} \backslash P$ of such an annular neighborhood $N$, equipped with the metric induced from $h$, is complete as a metric space.

In Case (2), no neighborhood of the puncture, equipped with the metric induced by $h$, is complete as a metric space. To obtain complete neighborhoods of the puncture, one adds to the surface $S_{\mathfrak{g}} \backslash P$ a simple closed curve in such a way that the surface at the puncture becomes a surface with boundary. The closed curve that is added is then a closed geodesic for the completed surface. The leaves of $F$ abut perpendicularly on that closed geodesic.

The hyperbolic metric on the surface $S_{\mathfrak{g}} \backslash P$ is complete if and only if all the punctures of $S_{\mathfrak{g}} \backslash P$ are of the type described in Case 1.

\section{Shift parameters for a triangulation of the sphere with three distinguished points}

In this section, we consider the sphere $S=S^{2}$ with three distinguished points, equipped with a triangulation $\mu$. Up to homeomorphisms of the sphere that fix pointwise the three distinguished points, there are exactly four such triangulations, and they are represented in Figure 6. Any such triangulation has exactly three
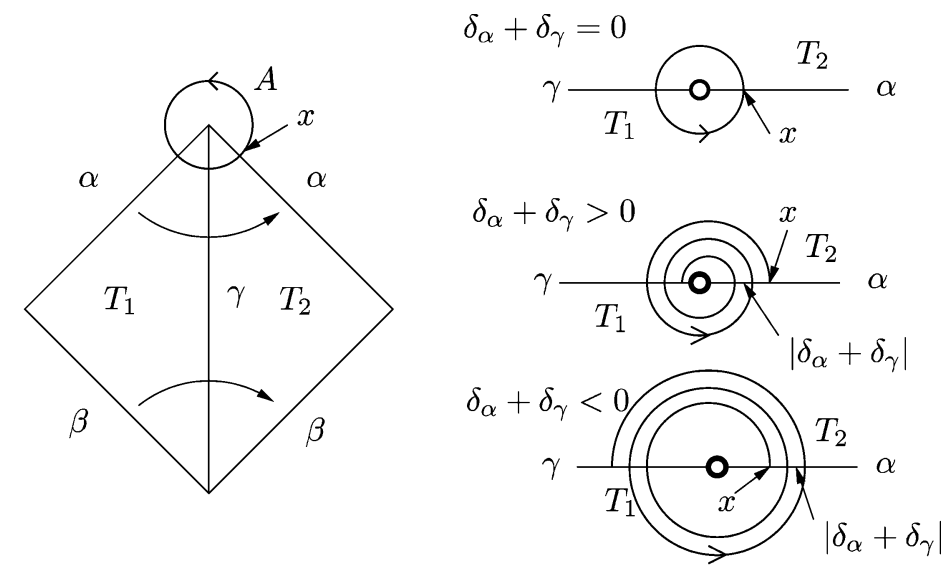

Figure 5. The surface $S$ is obtained by gluing the two triangles $T_{1}$ and $T_{2}$ according to the pattern indicated by the arrows in the figure to the left hand side. The type of the holonomy along the closed curve $A$ that surrounds the vertex is determined by the behaviour of a leaf of the horocyclic foliation starting at any point $x$, shown in the right hand side figure. This holonomy type is determined by the shifts $\delta_{\beta}$ and $\delta_{\gamma}$. More precisely, if $\left|\delta_{\beta}+\delta_{\gamma}\right|=0$, then the holonomy is parabolic and the corresponding vertex in the resulting surface is a cusp. Otherwise, the holonomy is hyperbolic and $\left|\delta_{\beta}+\delta_{\gamma}\right|$ is equal to the distance between two successive points of intersection of a leaf of the foliation with an edge of the triangulation 


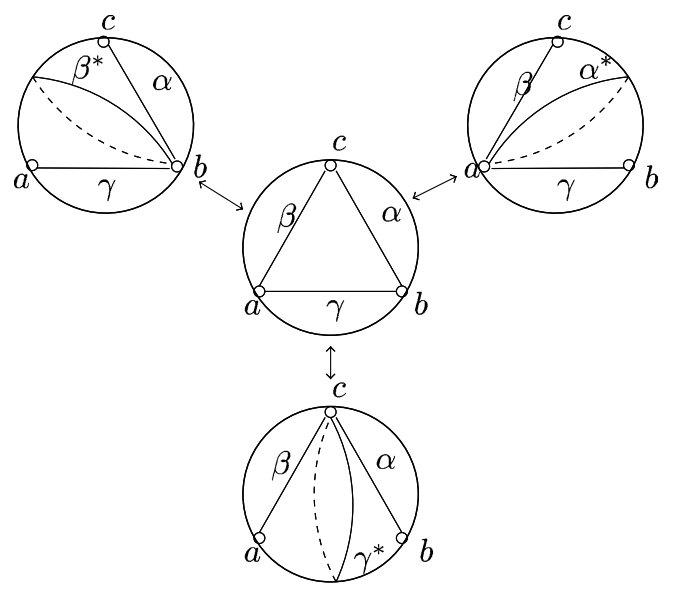

Figure 6. The four isotopy types of triangulations with 3 vertices of the sphere $S^{2}$, together with the flips between them
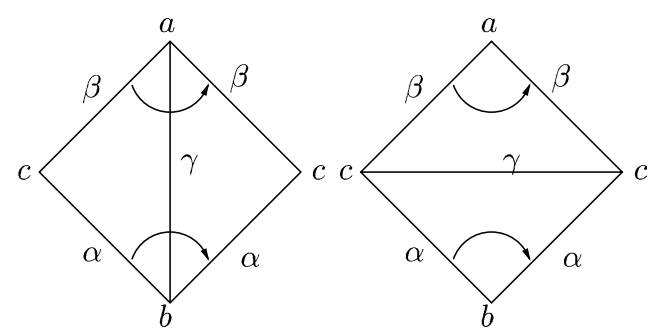

Figure 7. The combinatorics of the flip operation between the central triangulation in Figure 6 and the one to the bottom in that same figure

edges and two triangles, and there are three possibilities for the combinatorial type of a vertex $a$ in such a triangulation:

(1) exactly one half-edge abuts on $a$;

(2) exactly two half-edges abut on $a$ (and in that case, the two half-edges are necessarily half-edges of distinct edges of $\mu$ );

(3) four half-edges abut on $a$ (and in that case, exactly two of these half-edges are half-edges of distinct edges of $\mu$, and the other two are half-edges of the third edge of $\mu$ ).

Furthermore, there are exactly three flip operations that can be performed between these four triangulations; they are represented by the double arrows in Figure 6.

4.1. Symmetric and nonsymmetric triangulation. We shall consider hyperbolic structures on the sphere with its three distinguished points. For each such hyperbolic structure, we can realize the triangulation $\mu$ as an ideal triangulation. To analyze such an ideally triangulated hyperbolic metric, we can start with two ideal triangles and glue them together along their edges in such a way as to obtain 
(topologically) a three-punctured sphere. There are two combinatorially distinct gluing patterns between the edges of two ideal triangles that give a three-punctured sphere. One of them is represented by the central triangulation of Figure 6 and the other one is represented by the three other triangulations of that figure. We shall call these types of triangulations a symmetric and a nonsymmetric triangulation respectively.

Let $a, b, c$ be the three distinguished points of the sphere $S$, let $g$ be a hyperbolic structure on $(S,\{a, b, c\})$ and let $\mu$ be a triangulation of this pair. We need to have a reference orientation on the boundary components of this hyperbolic surface, and we equip each boundary component with the positive orientation induced from the orientation of the surface. We recall that this positive boundary orientation is defined in such a way that for any point $x$ on the boundary, the ordered pair consisting of a vector at $x$ which is tangent to the boundary and which points in the positive direction, followed by a tangent vector at $x$ pointing in the direction of the interior of the surface, constitutes a direct basis of the tangent space of the surface $S$ at $x$. In the next proposition, the terms "positive direction" and "negative direction" applied to a geodesic on the surface that spirals around a boundary component refer to this reference orientation.

We shall use the following notations. For any edge $e$ of $\mu$, we shall denote by $\delta_{e}$ the shift coordinate induced by the hyperbolic structure $g$ on that edge. Let $v$ be an element of the vertex set $\{a, b, c\}$. If $v$ becomes a boundary component when $(S,\{a, b, c\})$ is equipped with the hyperbolic structure $g$, we let $l_{g}(v)$ denote the length of that boundary component, and if $v$ becomes a cusp, we set $l_{g}(v)=0$.

With these notations, we have the following:

Proposition 4.1. Let $g$ be a hyperbolic structure on the sphere with three distinguished points $(S,\{a, b, c\})$. The holonomy type of a vertex and the length of that vertex depend only on the shift coordinates on the edges of $\mu$ that abut on that vertex. To be more precise, let us consider a vertex $a$. Then, there are three cases:

Case 1. Exactly two edges $\beta$ and $\gamma$ of $\mu$ have one of their ends on the vertex a. In that case, we have

$$
l_{g}(a)=\left|\delta_{\beta}+\delta_{\gamma}\right| .
$$

Furthermore, the holonomy at the vertex $a$ is hyperbolic if $\left|\delta_{\beta}+\delta_{\gamma}\right| \neq 0$ and parabolic if $\left|\delta_{\beta}+\delta_{\gamma}\right|=0$. In the case where the holonomy is hyperbolic, the geodesics associated to $\beta$ and $\gamma$ spiral around the simple closed geodesic that is homotopic to the vertex $a$ in the positive direction if $\delta_{\beta}+\delta_{\gamma}<0$ and in the negative direction if $\delta_{\beta}+\delta_{\gamma}>0$.

Case 2. Exactly four half-edges of $\mu$ abut on the vertex a, and two of these halfedges are half-edges of a single edge of $\mu$. Let $\alpha$ denote that single edge of $\mu$ and let $\beta$ and $\gamma$ denote the other two edges that abut (from one side) on a. In that case, we have

$$
l_{g}(a)=\left|\delta_{\beta}+\delta_{\gamma}+2 \delta_{\alpha}\right| .
$$


Furthermore, the holonomy at the vertex a is hyperbolic if $\left|\delta_{\beta}+\delta_{\gamma}+2 \delta_{\alpha}\right| \neq 0$ and parabolic if $\left|\delta_{\beta}+\delta_{\gamma}+2 \delta_{\alpha}\right|=0$. In the case where the holonomy is hyperbolic, the edges of $\mu$ spiral around the boundary closed geodesic that is homotopic to the vertex $a$ in the positive direction if $\delta_{\beta}+\delta_{\gamma}+2 \delta_{\alpha}<0$, and in the negative direction if $\delta_{\beta}+\delta_{\gamma}+2 \delta_{\alpha}>0$.

Case 3. There is a unique edge $\beta$ of $\mu$ that abuts on the vertex $a$. In that case,

$$
l_{g}(a)=\left|\delta_{\beta}\right| .
$$

Furthermore, the holonomy at the vertex $a$ is hyperbolic if $\left|\delta_{\beta}\right| \neq 0$ and parabolic if $\left|\delta_{\beta}\right|=0$. In the case where the holonomy is hyperbolic, the spiraling around the boundary simple closed geodesic homotopic to the vertex $a$ is in the positive direction if $\delta_{\beta}<0$, and in the negative direction if $\delta_{\beta}>0$.

Proof. In each case, we shall obtain the information on the holonomy at the vertex $a$ and about the length of that vertex by taking an appropriate fundamental domain for the surface in the upper half-plane model of $\llbracket^{2}$ and examining the isometries of $\mathbb{Q}^{2}$ that perform the pairing of the sides of that fundamental domain.

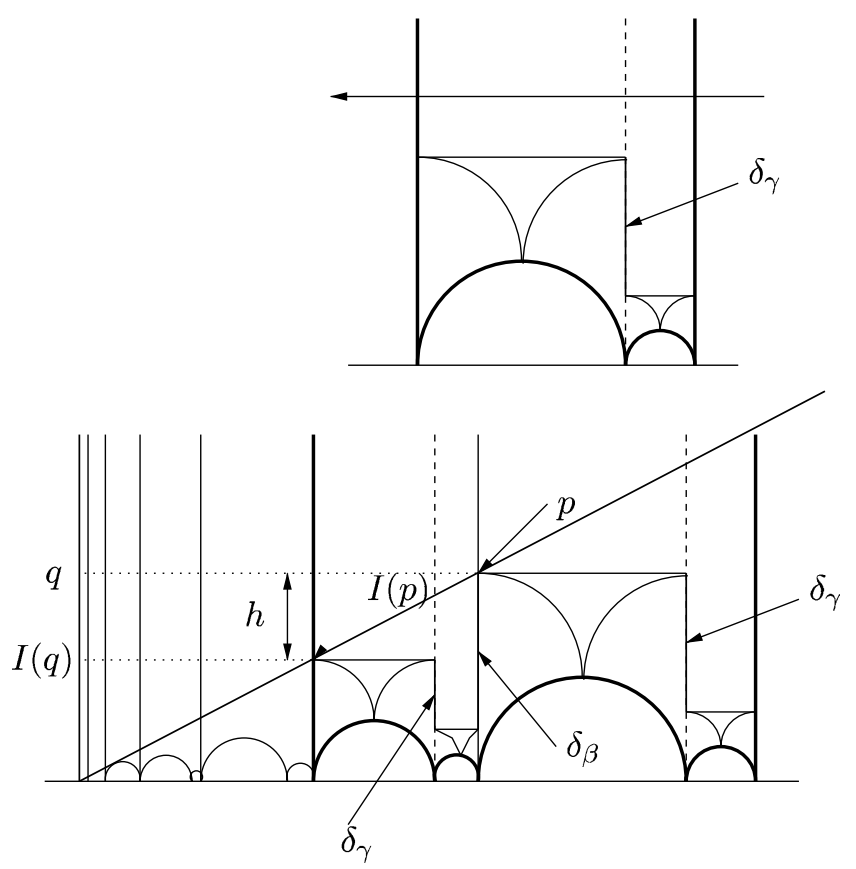

Figure 8. This figure refers to Case 1 of Proposition 4.1 where there are two edges of $\mu$ abutting on the vertex $a=\infty$ and where the holonomy around that vertex is hyperbolic. The upper picture represents a quadrilateral $Q$ which is a fundamental domain of the group of deck transformations of the covering. The lower picture represents the successive images of $Q$ by the action of the cyclic group generated by $I$. These images accumulate on the geodesic in $\mathbb{Q}^{2}$ that is invariant by the hyperbolic isometry $I$. We have represented a point $p$ on the boundary of the quadrilateral $Q$ and its image $I(p)$. The whole orbit of the point $p$ by the group generated by $I$ is situated on a hypercycle.

The two endpoints of the hypercycle are the two endpoints of the geodesic invariant by $I$ 
In particular, we can see from this picture of the fundamental domain the isometry that gives the holonomy type at $a$. Let $I$ be this isometry. We shall study below the nature of the isometry $I$ in each case.

We first consider the pattern corresponding to Case 1. This is represented in Figure 8, in which the upper picture is a fundamental domain $Q$ of the threepunctured sphere. This fundamental domain $Q$ is a quadrilateral that is the union of two ideal triangles $T_{1}$ and $T_{2}$ glued along a common edge. Without loss of generality, we can assume that one of their common vertices, $a$, is the point $\infty$. The horizontal segment with an arrow in the upper picture is a lift of a simple closed curve around the vertex $a$ in the quotient surface, and we study the holonomy of this closed curve. In the case drawn in the figure, the shift $\delta_{\gamma}$ on the edge that is common to the two ideal triangles is negative (i.e. we have a right shear). The bottom picture in Figure 8 represents two copies of $Q$ that are glued along a common edge $\beta$ with a positive shift $\delta_{\beta}$. The deck transformation $I$ that sends the quadrilateral $Q$ to the adjacent quadrilateral situated to its left must send the distinguished point $p$ on a boundary edge of $Q$ to the distinguished point $I(p)$ on the corresponding boundary edge of $Q$ as shown in that figure, since these two distinguished points project on the same point on the surface. The deck transformation $I$ is a representative of the holonomy at the vertex $a$. In the case considered, we have $\delta_{\beta}+\delta_{\gamma}<0$ and the isometry $I$ is hyperbolic. In the case where $\delta_{\beta}+\delta_{\gamma} \neq 0$, we choose coordinates in $\mathbb{T}^{2}$ so that the origin 0 of the real axis is the intersection point of the Euclidean line joining the points $p$ and $I(p)$ (this line is a hypercycle in the sense of hyperbolic geometry) with the $x$-axis. Then the isometry $I$ is of the form $z \mapsto e^{-h} z$, where $h=\left|\delta_{\beta}+\delta_{\gamma}\right|$ is the translation distance of that isometry. Note that in the case considered (i.e. the case where $\delta_{\beta}+\delta_{\gamma} \neq 0$ ), the point $p$ together with its image $I(p)$ allow us to determine geometrically the position of the geodesic that is invariant by the isometry $I$.

The case where the isometry $I$ is parabolic corresponds to $\delta_{\beta}+\delta_{\gamma}=0$. In that case, $p$ and its images are situated on a horocycle centered at $\infty$.

Note that if a vertex $a$ of $S$ is in the situation of Case 1, then the triangulation $\mu$ is symmetric (in the sense defined in Sect. 4.1), which implies that we have the following formulae for the lengths of the three vertices $a, b$ and $c$ of $S$ :

$$
\forall g \in \mathscr{T}(S), \quad l_{g}(a)=\left|\delta_{\beta}+\delta_{\gamma}\right|, \quad l_{g}(b)=\left|\delta_{\alpha}+\delta_{\gamma}\right|, \quad l_{g}(c)=\left|\delta_{\alpha}+\delta_{\beta}\right|,
$$

where $l_{g}(a)=0$ if and only if the holonomy type of $a$ is parabolic.

We now study the sense of the spiraling. This is best seen by looking at Figure 8. Recall our conventions on the sign of the shifts and on the orientation of the boundary components of $S$. They imply that when the three shifts are positive, the half-edges of $\mu$ spiral around the boundary components in the sense opposite to that of the reference orientation on that boundary component, induced from the orientation of the surface. This can be seen in Figure 8 where the geodesic line $(0, \infty)$, joining $q$ and $I(q)$, which is on the boundary of the universal cover of the hyperbolic surface and which is invariant by the isometry $I$, projects to a boundary geodesic on the quotient surface. The orientation of the geodesic line $(0, \infty)$ induced from the orientation of the surface is from top to bottom. The sequence of vertical geodesics that accumulate on the geodesic line $(0, \infty)$ are lifts 
of the edges of $\mu$ that spiral around the boundary closed geodesic which is the image of the line $(0, \infty)$ in the quotient surface. All these vertical geodesics have the vertex $\infty$ in common with the geodesic line $(0, \infty)$, and the accumulation is the result of applying a covering translation of the form $z \mapsto \lambda z$ with $0<\lambda<1$. The spiraling along the image closed geodesic in the quotient hyperbolic surface is in the direction induced from the orientation from 0 to $\infty$ on the line $(0, \infty)$, which indeed corresponds to the negative orientation on the image closed geodesic.

Now we consider Case 2. A fundamental domain for the surface is represented in Figure 9. A simple closed curve around the vertex $a$ has four intersection points with the edges of the ideal triangulation. Note that if such a closed curve is chosen in minimal intersecting position with respect to $\mu$, then its intersection with each ideal triangle has two components. The upper picture in Figure 9 represents, in the upper half-space $\mathbb{W}^{2}$, the ideal triangles that the lift of this curve passes through, with the point $a$ being at $\infty$. This lift is represented as a horizontal line with an arrow, as was done in Figure 8 that corresponds to the preceding case. The shifts that are associated to the four edges that the curve crosses are (from left to right) $\delta_{\beta}, \delta_{\alpha}, \delta_{\gamma}, \delta_{\alpha}, \delta_{\beta}$. To see this, note that in the ideal triangulation considered of the sphere with three cusps or boundary components, the edge $\alpha$ separates the surface, and $\delta_{\alpha}$ represents the shift that identifies the two components of the surface $S$ cut along $\alpha$. Thus, when we follow the simple closed curve homotopic to $a$, we cross two times the edge $\alpha$, and at each time the shift is $\delta_{\alpha}$. In the lower picture, we have represented the image of the upper picture under the action of the group generated by the isometry $I$ that represents the holonomy at the vertex $a$. In the case drawn, this holonomy is hyperbolic, and it is represented by the map $z \mapsto e^{-h} z$ where
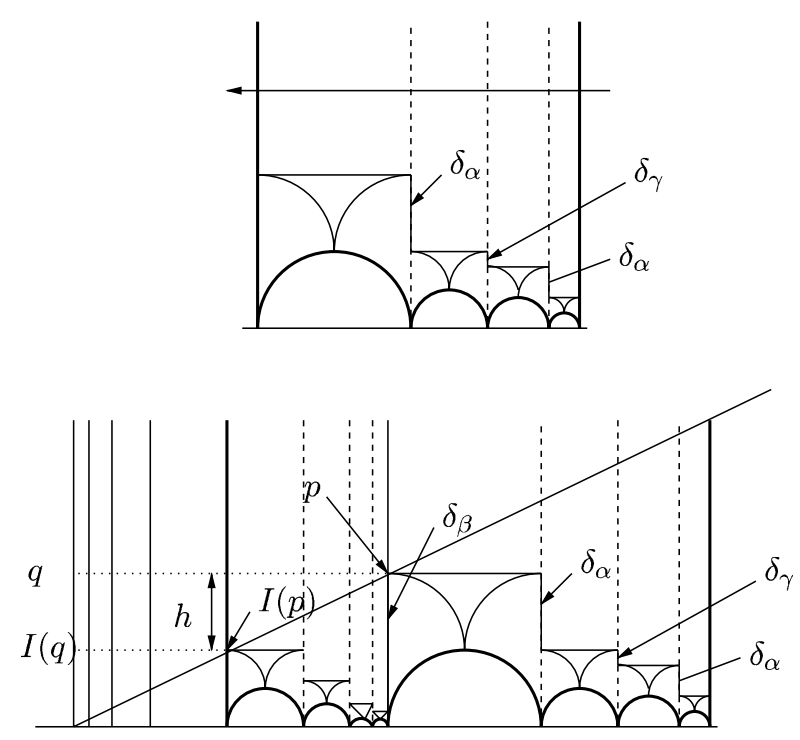

Figure 9. The holonomy at a vertex in the case where there are four half-edges abutting on that vertex (Case 2 of Proposition 4.1) 

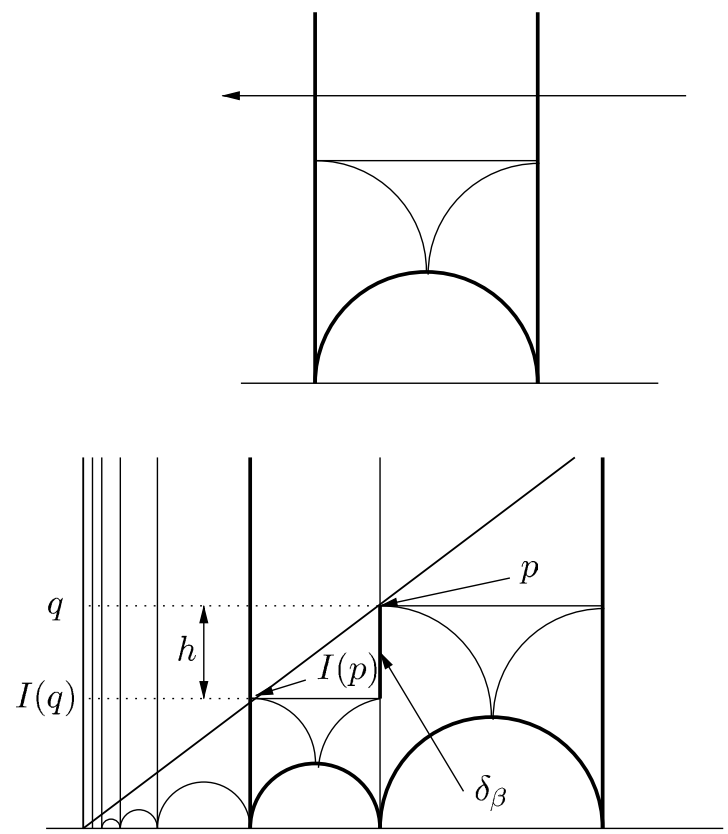

Figure 10. The holonomy in the case where there is only one edge abutting on a vertex (Case 3 of Proposition 4.1)

$h=\left|\delta_{\beta}+2 \delta_{\alpha}+\delta_{\gamma}\right|$. The rest of the analysis is similar to the one of the previous cases.

The situation in Case 3 is represented in Figure 10. It can be treated in the same way as the two other cases. (As a matter of fact, this is the simplest case.)

4.2. Signed length. It is convenient to introduce the notion of signed length $\widetilde{l_{g}}(a)$ of a distinguished point $a$ of the sphere with three distinguished points equipped with an ideal triangulation $\mu$ and a hyperbolic structure $g$. (The distinguished point is, as usual, either a boundary component or a cusp of the hyperbolic structure $g$.) This length is nonzero if and only if the vertex is a boundary component, and its sign depends on the direction of the spiraling of the edges of $\mu$ around the boundary component $a$. More precisely, following the notations of Proposition 4.1, we set, for a given vertex $a$,

$$
\widetilde{l}_{g}(a)=\left\{\begin{array}{l}
l_{g}(a) \text { if the spiraling is in the negative direction } \\
-l_{g}(a) \text { if the spiraling is in the positive direction } \\
0 \text { if } a \text { is a cusp. }
\end{array}\right.
$$

Using the notion of signed length, the formulae given in Proposition 4.1 become respectively

$$
\left\{\begin{array}{l}
\widetilde{l}_{g}(a)=\delta_{\beta}+\delta_{\gamma} \\
\widetilde{l}_{g}(a)=\delta_{\beta}+\delta_{\gamma}+2 \delta_{\alpha} \\
\widetilde{l}_{g}(a)=\delta_{\beta}
\end{array}\right.
$$




\section{The unfolded Teichmüller space associated to a triangulation with three vertices of the sphere}

Let $\mu$ be a triangulation of the sphere $S=S^{2}$ with three distinguished points. In this section, we show that the shift coordinates on the edges of $\mu$ give a parametrization of the unfolded Teichmüller space $\widetilde{T}(S)=\widetilde{T}(S, P)$ by $\mathbb{R}^{3}$. We recall that the elements of $\widetilde{T}(S)$ are isotopy classes of signed hyperbolic structures. In this parametrization, the orientation on the geodesic boundary components is induced by the direction of spiraling of the edges of $\mu$ realized as an ideal triangulation around these boundary components.

We shall analyze several features of the parametrization of $\widetilde{T}(S)$ by shift coordinates and we shall give a description of $\mathscr{T}(S)$ as a cone over an octahedron in $\mathbb{R}^{3}$. Each open simplex of the octahedron corresponds to a special kind of behaviour at the vertices of $\mu$.

We call the Teichmüller space of a given surface with orientations specified on its boundary components, a special Teichmüller space.

Theorem 1. The shift coordinates on the edges of $\mu$ parametrize the unfolded Teichmüller space $\widetilde{T}(S)$ by $\mathbb{R}^{3}$. This parametrization gives a natural description of $\widetilde{T}(S)$ as the cone over an octahedron. Each open simplex of the octahedron corresponds to a special Teichmüller space embedded in $\widetilde{T}(S)$. The origin $O$ of $\mathbb{R}^{3}$ parametrizes the special Teichmüller space of the sphere with three cusps (which is indeed reduced to one point). Furthermore, the unfolded Teichmüller space has a canonical projection onto an augmented Teichmüller space of the pair of pants, that is, the union of the Teichmüller space of the pair of pants with the various Teichmüller spaces of the generalized pair of pants with one, two or three cusps on their boundary.

In the rest of this section, we prove this theorem. In fact, we shall give a more complete description of $\widetilde{T}(S)$ and of its subspaces than the one in the statement of the theorem.

We note that although our definition of the unfolded Teichmüller space does not make use of the triangulation $\mu$, the parametrization of that space by $\mathbb{R}^{3}$ depends on $\mu$, and the structure of that space as a combinatorial object and, a priori, its topology, could depend on $\mu$. We shall sometimes denote by $\mathscr{T}^{\mu}(S)$ the unfolded Teichmüller space equipped with its parametrization by shift coordinates on the edges of $\mu$. We shall study below the dependence of this combinatorial structure on the choice of $\mu$.

We treat in detail the case where $\mu$ is a symmetric triangulation. (Recall that this is the case of the triangulation represented at the center of Figure 6). In this case, the picture of the combinatorial structure of the unfolded Teichmüller space $\mathscr{T}^{\mu}(S)$ is given in Figure 11.

We use the notations that we introduced before, in which $\alpha, \beta, \gamma$ are the three edges of $\mu$, and $\delta_{\alpha}, \delta_{\beta}, \delta_{\gamma}$ are the corresponding shifts.

The Euclidean space $\mathbb{R}^{3}$, with coordinates $\delta_{\alpha}, \delta_{\beta}, \delta_{\gamma}$, is subdivided into eight conical regions by the three hyperplanes defined by the equations $\delta_{\alpha}=0, \delta_{\beta}=0$ and $\delta_{\gamma}=0$. In Figure 11, we have represented two octahedra in $\mathbb{R}^{3}$, which we call 


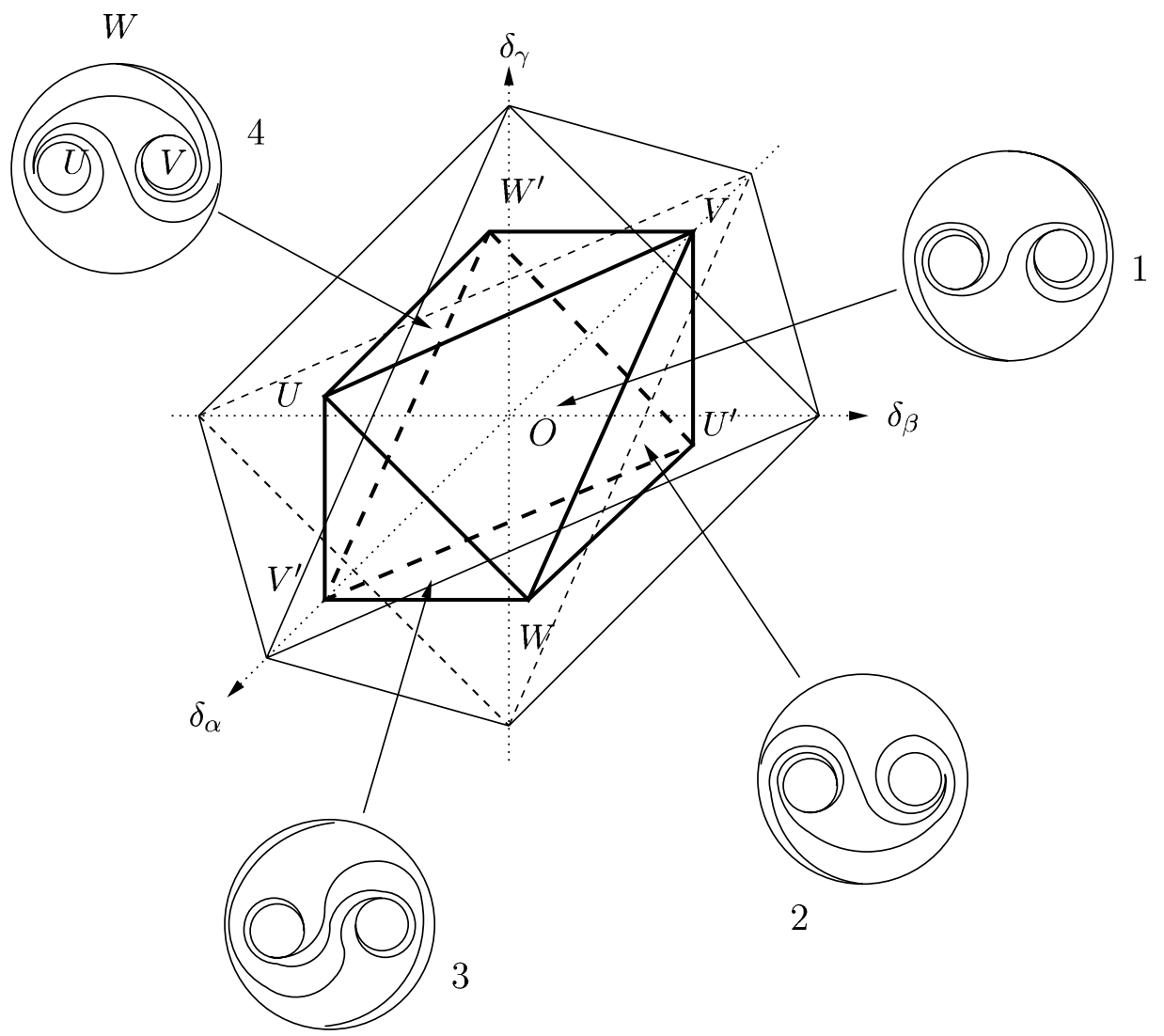

Figure 11. The two-dimensional faces of the large octahedron $\mathcal{O}^{\prime}$ (whose edges are drawn in light lines) are the intersections of that octahedron with the eight standard quadrants of $\mathbb{R}^{3}$. The small octahedron $\mathcal{O}$ (drawn in bold lines) is the octahedron dual to $\mathcal{O}^{\prime}$. Each of the eight cones over the open two-dimensional faces of $\mathcal{O}$ parametrizes the Teichmüller space $\mathscr{T}\left(S_{0,3,0}\right)$ of the sphere with three boundary components. Each of these Teichmüller space $\mathscr{T}\left(S_{0,3,0}\right)$ is characterized by a given way of spiraling of the edges of $\mu$ around the boundary components. Likewise, the cones over the lowerdimensional simplices parametrize Teichmüller spaces of the sphere in which one or more distinguished points are punctures, the rest of the distinguished points being boundary components

$\mathcal{O}$ and $\mathcal{O}^{\prime}$. The larger one, $\mathcal{O}^{\prime}$, is the octahedron whose two-simplices are the intersections of these eight regions with the hyperplanes of equations $e_{1} \delta_{\alpha}+e_{2} \delta_{\beta}+$ $e_{3} \delta_{\gamma}=1$, where each equation is associated to a triple $\left(e_{1}, e_{2}, e_{3}\right)$, where $e_{1}, e_{2}$ and $e_{3}$ take their values in $\{0,1\}$. The vertices of the octahedron $\mathcal{O}$ are the barycenters of the two-dimensional faces of the octahedron $\mathcal{O}^{\prime}$, that is, the points $U=$ $(1 / 3,-1 / 3,1 / 3), V=(-1 / 3,1 / 3,1 / 3), W=(1 / 3,1 / 3,-1 / 3)$ and the points $U^{\prime}, V^{\prime}, W^{\prime}$ that are symmetric to $U, V, W$, respectively with respect to $O$. Note that, geometrically, the octahedron $\mathcal{O}$ is obtained from a cube by chopping off two pieces bounded by the two triangular faces $U V W$ and $U^{\prime} V^{\prime} W^{\prime}$ of that octahedron.

The octahedron $\mathcal{O}$ (and, likewise, the octahedron $\mathcal{O}^{\prime}$ ), viewed as a simplicial complex, has $8+12+6=26$ distinct simplices. Each of the 26 positive cones 
over the interior of such a simplex, together with the origin $O$ of $\mathbb{R}^{3}$ (which make in total 27 objects), is a Teichmüller space of the sphere with three distinguished points where each of the distinguished points has been specified to be either a puncture or a boundary component, that is, a space whose elements are isotopy classes of hyperbolic structures on a fixed (generalized) pair of pants. Several of the Teichmüller spaces that occur in this collection are Teichmüller spaces of the same surface, and there are canonical identifications between them. Each of these spaces is specified by a certain way of spiraling of the edges of $\mu$ around the boundary components of the surface or, equivalently, by an orientation on each boundary geodesic.

Thus, the topology induced on the unfolded Teichmüller space $\widetilde{T}(S)$ by the shift coordinates on the edges of $\mu$ makes a continuous link between any two special Teichmüller spaces of generalized pairs of pants. This will be made precise below. Degenerate structures where the length of a simple closed curve is equal to 0 are elements of the unfolded Teichmüller space $\mathscr{T}^{\mu}(S)$, and they also appear as boundary structures of the various special Teichmüller spaces of pairs of pants. Recall that these structures also appear in complex analysis as surfaces with nodes, on the boundary of Teichmüller space.

More precisely, the space $\mathbb{R}^{3}$ parametrizes an unfolded Teichmüller space which is the union of $26+1=27$ subspaces, each of which can be identified with a Teichmüller space in the usual sense. These subspaces can be described as follows:

- Each positive cone over one of the eight open two-dimensional faces of the octahedron $\mathcal{O}$ is a special Teichmüller space of a pair of pants with three boundary geodesics. Such a Teichmüller space is distinguished by the fact that the three distinguished points are chosen to be boundary components, and it is distinguished from the two other congruent spaces by a specific sense of spiraling for the edges of the ideal triangulation $\mu$ around the boundary geodesics. Four of these spiraling behaviours are represented in Figure 11.

- Each positive cone over an open one-dimensional face of the octahedron $\mathcal{O}$ is a special Teichmüller space of a (generalized) pair of pants with one cusp and two boundary geodesics. There are 12 such special Teichmüller spaces, and each of them is specified by the three different choices of the cusp for the sphere with three distinguished points, and, for each such choice, by a specific sense of spiraling of the edges of the ideal triangulation $\mu$ around the two boundary geodesics of the surface.

- Each positive cone over a vertex of the octahedron $\mathcal{O}$ is the Teichmüller space of a generalized pair of pants with two cusps and one boundary geodesic. There are six such special Teichmüller spaces, each of them corresponding to a special choice of the boundary component among the three distinguished points of the sphere, and, for each such choice, by the sense of spiraling of the edges of the ideal triangulation $\mu$ around that boundary geodesic.

- Finally, the origin $O$ corresponds to the Teichmüller space of the sphere with three cusps.

Note that for each of the eight cones over the two-dimensional faces of $\mathcal{O}$, the various spiraling behaviours around the geodesic boundary components are deter- 
mined by linear inequalities in the shift parameters on the edges of the ideal triangulation $\mu$. For instance, the case labelled 1 in Figure 11 (that is, the cone over the triangle $U V W$ ) corresponds to the system of inequalities

$$
\left\{\begin{array}{l}
\delta_{\beta}+\delta_{\gamma}>0 \\
\delta_{\alpha}+\delta_{\gamma}>0 \\
\delta_{\alpha}+\delta_{\beta}>0
\end{array}\right.
$$

Case 2 (the cone over the triangle $U^{\prime} V W$ ) corresponds to

$$
\left\{\begin{array}{l}
\delta_{\beta}+\delta_{\gamma}>0 \\
\delta_{\alpha}+\delta_{\gamma}<0 \\
\delta_{\alpha}+\delta_{\beta}>0
\end{array}\right.
$$

Case 3 (the cone over the triangle $U V^{\prime} W$ ) corresponds to

$$
\left\{\begin{array}{l}
\delta_{\beta}+\delta_{\gamma}>0 \\
\delta_{\alpha}+\delta_{\gamma}>0 \\
\delta_{\alpha}+\delta_{\beta}<0 .
\end{array}\right.
$$

Case 4 (the cone over the triangle $U V W^{\prime}$ ) corresponds to

$$
\left\{\begin{array}{l}
\delta_{\beta}+\delta_{\gamma}<0 \\
\delta_{\alpha}+\delta_{\gamma}>0 \\
\delta_{\alpha}+\delta_{\beta}>0
\end{array}\right.
$$

5.1. The type of the horocyclic foliation. In Figure 12, the triangular face $U V W$ is subdivided into four smaller triangles. The fact that a point of $\mathscr{T}^{\mu}(S)$ which is in

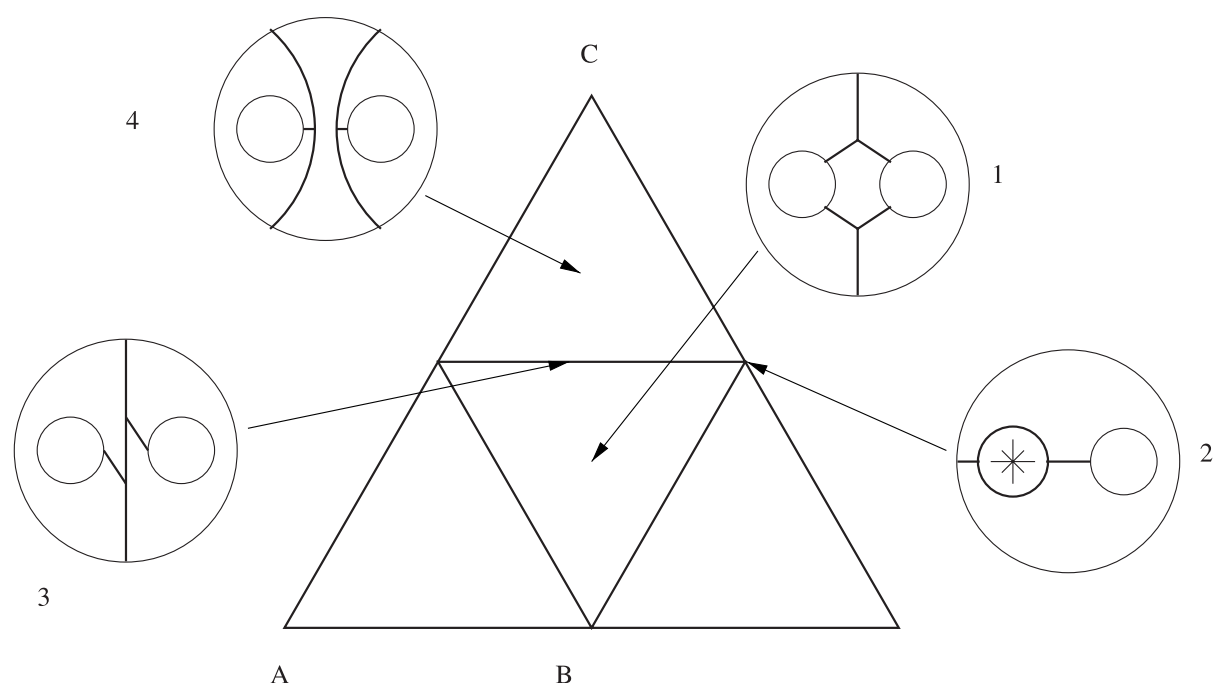

Figure 12. This figure represents the patterns of the singular graphs of the horocyclic foliations for the various hyperbolic structures representing points in the cone over the face $U V W$ of the octahedron (1) The star indicates a cusp 
the cone over the face $U V W$ belongs to one of the various cones over the smaller triangles represented in this figure depends on the type of the horocyclic foliation $F_{\mu}(g)$ of a hyperbolic structure $g$ representing this point. More precisely, this fact depends on the behaviour of the singular graph of the measured foliation $F_{\mu}^{\prime}(g)$ obtained from $F_{\mu}(g)$ by collapsing each non-foliated region onto a tripod (that is, a graph consisting in a singular point with three segments contained in the leaves starting at that point). Note that the measured foliation $F_{\mu}^{\prime}(g)$ is well defined up to isotopy. The singular graph of a measured foliation on a pairs of pants which is transverse to the boundary is the union of the leaves that start at singular points. The various topological types of singular graphs are represented in Figure 12. Note that for any hyperbolic structure $g$ representing a point in the interior of the cone over $U V W$, the horocyclic foliation $F_{\mu}(g)$ is transverse to the boundary of the pair of pants.

If $g$ is any hyperbolic structure on the pair of pants $S$ equipped with the ideal triangulation $\mu$, the associated horocyclic measured foliation $F_{\mu}(g)$ is transverse to the ideal triangulation $\mu$. We already saw that any measured foliation transverse to $\mu$ induces a set of shift coordinates on the edges of $\mu$. Notice that the shift parameter on an edge of $\mu$ induced by a hyperbolic structure $g$ is equal to the shift parameter on that edge induced by the horocyclic measured foliation $F_{\mu}(g)$.

Consider again the division of the triangular face $U V W$ into four smaller triangles (Figure 12). The three segments in the interior of the face that define this division correspond to hyperbolic metrics $g$ on the sphere with three distinguished points, $a, b, c$ that are defined in terms of lengths of the closed geodesics representing these points by the equalities $l_{g}(a)=l_{g}(b)+l_{g}(c), l_{g}(b)=l_{g}(a)+$ $l_{g}(c)$ and $l_{g}(c)=l_{g}(a)+l_{g}(b)$, respectively. (Remember that, by convention, a cusp is considered as a geodesic of length 0 .) Note that one can write equivalent equations in terms of transverse measures of the boundary components, with respect to the horocyclic foliations.

5.2. Identifications between special Teichmüller spaces. There are natural identifications between special Teichmüller spaces contained in the unfolded Teichmüller space. Namely, let $\mathscr{T}_{1}$ and $\mathscr{T}_{2}$ be two Teichmüller spaces of $S$ that correspond to hyperbolic structures of the same type (meaning that any distinguished point of the surface $S$ is a cusp for both structures or a boundary component for both structures). Then, it is natural to identify any two points in $\mathscr{T}_{1}$ and $\mathscr{T}_{2}$ whenever they are represented by the same hyperbolic structure. Note that this amounts to forgetting the sign of the hyperbolic structures. This gives a homeomorphism $\mathscr{T}_{1} \rightarrow \mathscr{T}_{2}$. Such a homeomorphism is described in a simple manner using the shift parameters. For instance it is easy to see that if the two spaces $\mathscr{T}_{1}$ and $\mathscr{T}_{2}$ are of maximal dimension and are represented in $\mathbb{R}^{3}$ by two three-dimensional cones that share a common two-dimensional face, then the identification is a (non-orthogonal) symmetry that fixes that face. Specifically, consider the two Teichmüller spaces that are parametrized by the three-dimensional cones over the open faces $U V W$ and $U V W^{\prime}$ of $\mathcal{O}$. Then, the natural identification between these two subspaces is the unique linear involution that preserves the two-dimensional face $O U V$ and that sends $W$ to $W^{\prime}$. The other identifications between the 3-dimensional spaces are compositions of such symmetries. 
Each of the eight three-dimensional special Teichmüller spaces is parametrized by a cone that is defined by three inequalities. Each two such Teichmüller spaces $\mathscr{T}_{1}$ and $\mathscr{T}_{2}$ that share a common 2-dimensional face are defined by three inequalities, two of which are identical and the third one being different. For instance, the cones over the faces $U V W$ and $U V W^{\prime}$ are defined respectively by the systems of equations

$$
\left\{\begin{array}{l}
\delta_{\alpha}+\delta_{\beta}>0 \\
\delta_{\alpha}+\delta_{\gamma}>0 \\
\delta_{\beta}+\delta_{\gamma}>0
\end{array}\right.
$$

and

$$
\left\{\begin{array}{l}
\delta_{\alpha}+\delta_{\beta}<0 \\
\delta_{\alpha}+\delta_{\gamma}>0 \\
\delta_{\beta}+\delta_{\gamma}>0
\end{array}\right.
$$

Therefore, a point in $\mathscr{T}_{1}$ whose coordinates are $\left(\delta_{\alpha}, \delta_{\beta}, \delta_{\gamma}\right)$ is identified with the point in $\mathscr{T}_{2}$ whose coordinates $\left(\delta_{\alpha}^{\prime}, \delta_{\beta}^{\prime}, \delta_{\gamma}^{\prime}\right)$ satisfy

$$
\left\{\begin{array}{l}
\delta_{\alpha}^{\prime}+\delta_{\beta}^{\prime}=-\delta_{\alpha}-\delta_{\beta} \\
\delta_{\alpha}^{\prime}+\delta_{\gamma}^{\prime}=\delta_{\alpha}+\delta_{\gamma} \\
\delta_{\beta}^{\prime}+\delta_{\gamma}^{\prime}=\delta_{\beta}+\delta_{\gamma}
\end{array}\right.
$$

or equivalently

$$
\left\{\begin{array}{l}
\delta_{\alpha}^{\prime}=-\delta_{\beta} \\
\delta_{\beta}^{\prime}=-\delta_{\alpha} \\
\delta_{\gamma}^{\prime}=\delta_{\alpha}+\delta_{\beta}+\delta_{\gamma} .
\end{array}\right.
$$

With this, we can easily check that the symmetry $s$ that exchanges the two spaces is induced by the linear map of $\mathbb{R}^{3}$ whose matrix in the canonical basis is

$$
\left(\begin{array}{ccc}
0 & -1 & 0 \\
-1 & 0 & 0 \\
1 & 1 & 1
\end{array}\right) .
$$

This matrix has $\left(\begin{array}{c}1 \\ -1 \\ 0\end{array}\right)$ and $\left(\begin{array}{l}0 \\ 0 \\ 1\end{array}\right)$ as eigenvectors with eigenvalue 1 and $\left(\begin{array}{c}1 \\ 1 \\ -1\end{array}\right)$ as eigenvector with eigenvalue -1 .

In other words, $s$ is the linear map that fixes the plane $\delta_{\alpha}+\delta_{\beta}=0$ (that is, the common face to the two cones) and that acts as a symmetry along the line $\left(W W^{\prime}\right)$.

We denote by $\mathscr{G}$ the group of linear homeomorphisms of $\mathbb{R}^{3}$ that is generated by all the natural identifications between the various special Teichmüller spaces. The group $\mathscr{G}$ is finite. The quotient of $\widetilde{T}(S)$ by this group is an augmented Teichmüller space of the pair of pants, that is, the union of the Teichmüller space of the pair of pants with the various Teichmüller spaces of the generalized pair of pants on its boundary.

5.3. The central symmetry. Let $P$ be a generalized hyperbolic pair of pants. The canonical involution $\iota$ on $P$ (cf. Sect. 2.6) preserves each boundary component of $P$, 
and if $P$ is equipped with an ideal triangulation $\mu$, then $\iota$ sends $\mu$ to an ideal triangulation $\bar{\mu}$ which spirals around each boundary component in the sense opposite to that of $\mu$. The set of all such maps $\iota$ on generalized hyperbolic pairs of pants is induced by a map which is defined on the unfolded Teichmüller space $\widetilde{T}(S)$ of the sphere with three distinguished points. In the space $\mathbb{R}^{3}$ of shift coordinates on the edges of $\mu$, this map is the symmetry with respect to the origin $O$. Note that the fixed point set of this involution of $\mathbb{R}^{3}$ is the origin as expected, since the sphere with three cusps coincides with its image by its order-two symmetry as points in the unfolded Teichmüller space $\widetilde{\mathscr{T}}(S)$.

\section{Changing the ideal triangulation}

The shift coordinates associated to an ideal triangulation $\mu$ on a sphere $S$ with three distinguished points define a homeomorphism $\eta$ between the unfolded Teichmüller space $\widetilde{T}(S)$ and $\mathbb{R}^{3}$, and therefore they provide the space $\widetilde{\mathscr{T}}(S)$ with a linear structure. Changing the ideal triangulation $\mu$ on $(S, P)$ to another triangulation $\mu^{\prime}$ induces a global coordinate change map $\eta^{\prime} \circ \eta^{-1}$ from $\mathbb{R}^{3}$ to itself.

Proposition 6.1. The map $\eta^{\prime} \circ \eta^{-1}$ is linear.

Proof. We start with a symmetric triangulation $\mu$ and we let $\left(\delta_{\alpha}, \delta_{\beta}, \delta_{\gamma}\right)$ be the shift coordinates on the edges $\mu$.

Let $\left(\delta_{\alpha}^{\prime}, \delta_{\beta}^{\prime}, \delta_{\gamma}^{\prime}\right)$ be the shift coordinates on the edges of a non-symmetric triangulation $\mu^{\prime}$ obtained from $\mu$ by a flip.

Let $g=\left(\widetilde{l_{g}}(a), \widetilde{l_{g}}(b), \widetilde{l_{g}}(c)\right) \in \mathbb{R}^{3}$ represent a hyperbolic structure, the tilde represent the signed lengths as defined in Sect. 4.2 above.

In the case of the triangulation $\mu$, we have

$$
\left\{\begin{array}{l}
\widetilde{l}_{g}(a)=\delta_{\beta}+\delta_{\gamma} \\
\widetilde{l}_{g}(b)=\delta_{\beta}+\delta_{\gamma} \\
\widetilde{l}_{g}(c)=\delta_{\beta}+\delta_{\gamma} .
\end{array}\right.
$$

In the case of the triangulation $\mu^{\prime}$, the formulae are

$$
\left\{\begin{array}{l}
\widetilde{l}_{g}(a)=\delta_{\beta}^{\prime}+\delta_{\gamma}^{\prime}+2 \delta_{\alpha}^{\prime} \\
\widetilde{l}_{g}(b)=\delta_{\beta}^{\prime} \\
\widetilde{l}_{g}(c)=\delta_{\beta}^{\prime} .
\end{array}\right.
$$

The natural identification between the two parameter spaces of the unfolded Teichmüller spaces given by the shifts on the edges of $\mu$ and $\mu^{\prime}$ is induced by the canonical identification between the signed hyperbolic structures that are parametrized by these spaces. At the level of the shift coordinates, the change in coordinates are given by the following linear formulae:

$$
\left\{\begin{array}{l}
\delta_{\alpha}^{\prime}=-\delta_{\alpha} \\
\delta_{\beta}^{\prime}=\delta_{\alpha}+\delta_{\beta} \\
\delta_{\gamma}^{\prime}=\delta_{\alpha}+\delta_{\gamma} .
\end{array}\right.
$$


Corollary 6.2. The shift coordinates provide the unfolded Teichmüller space of the sphere with three distinguished points with a linear structure which is independent of the choice of the triangulation $\mu$.

\section{Stretch lines and earthquakes on the unfolded Teichmüller space}

7.1. Stretch lines. Consider a surface $S_{\mathfrak{g}, \mathfrak{b}, \mathfrak{p}}$ equipped with an ideal triangulation $\mu$. We recall that a stretch line directed by $\mu$ in the Teichmüller space $\mathscr{T}\left(S_{\mathfrak{g}, \mathfrak{b}, \mathfrak{p}}\right)$ starting at (the equivalence class of) a hyperbolic $g$ is the map $t \mapsto g_{t}$ from $\mathbb{R}$ into $\mathscr{T}\left(S_{\mathfrak{g}, \mathfrak{b}, \mathfrak{p}}\right)$, where $g_{t}$ is the (equivalence class of the) hyperbolic structure whose horocyclic foliation $F_{\mu}\left(g_{t}\right)$ is obtained from the measured foliation $F_{\mu}(g)$ by multiplying its transverse measure by the factor $e^{t}$.

We shall say that the hyperbolic metric $g_{t}$ is obtained from $g$ by a $t$-stretch.

Proposition 7.1. The effect of a t-stretch multiplies the lengths of all the boundary geodesics of the surface by the factor $e^{t}$.

Proof. This follows from the fact that the leaves of the horocyclic foliation abut perpendicularly on the boundary geodesics, and that the transverse measure of this horocyclic foliation coincides with hyperbolic length on geodesic arcs that are perpendicular to the leaves of this foliation.

Now consider the special case where the surface is a pair of pants. Since a hyperbolic structure on a pair of pants is determined by the lengths of its three boundary components, any stretch line starting at a given point in the Teichmüller space of the pair of pants can be described as the line in that space obtained by changing the lengths of the boundary components of the surface by a common multiplicative factor. In particular, to obtain any stretch line in Teichmüller space, one can take an arbitrary ideal triangulation all whose bi-infinite edges spiral along the boundary geodesics of this pair of pants, and perform a stretch along that ideal triangulation. Any two such ideal triangulations give the same stretch line.

This is not true if we take an arbitrary surface $S_{\mathfrak{g}, \mathfrak{b}, \mathfrak{p}}$ equipped with a pair of pants decomposition. In other words, stretch lines starting at that surface which are defined after completing a given pair of pants decomposition into an ideal triangulation depend on the choice of the completion. We shall study precisely this phenomenon in Sect. 9 below. We shall see in some precise examples that if we start at some hyperbolic structure on a surface $S_{\mathfrak{g}, \mathfrak{b}, \mathfrak{p}}$ and perform stretches along
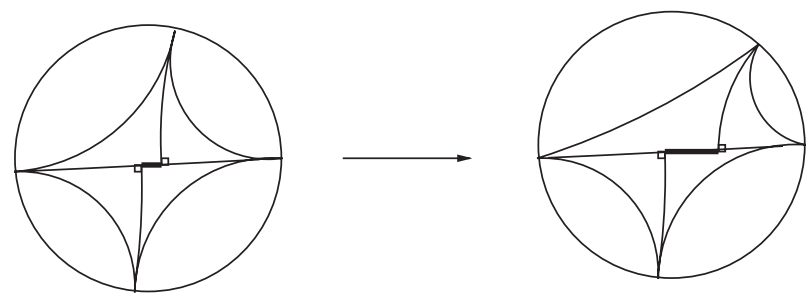

Figure 13. Varying a hyperbolic structure along a stretch line multiplies the shift parameters on the edges of the ideal triangulation by a constant factor 
ideal triangulations $\mu$ and $\mu^{\prime}$ that have different spiraling behaviour around geodesic curves of a given pair of pants decomposition, then these lines are distinct in the Teichmüller space of the surface, except of course at the starting point.

The next proposition states that we can define stretch lines on the unfolded Teichmüller space $\widetilde{T}(S)$ of a sphere with three distinguished points. We can send one of these lines in one cone over a simplex into a line in a cone over another simplex of the same dimension using elements of the group $\mathscr{G}$ of symmetries of $\widetilde{T}(S)$ that we described in Sect. 5.2 above.

In the shift parameters for $\mathscr{T}(S)$ associated to $\mu$, the stretch lines have a nice description, summarized in the following.

Proposition 7.2. Stretch lines can be defined on the unfolded Teichmüller space $\mathscr{T}(S)$. In the shift coordinates associated to an ideal triangulation $\mu, a$ stretch line directed by $\mu$ corresponds to a Euclidean ray starting at the origin. More precisely, a stretch line starting at a signed hyperbolic structure $g \in \widetilde{T}(S)$ is described, using the shift parameters, by

$$
t \mapsto\left(e^{t} \delta_{\alpha}(g), e^{t} \delta_{\beta}(g), e^{t} \delta_{\gamma}(g)\right), \quad t \in \mathbb{R} .
$$

With this definition, the flow induced on each special Teichmüller space in $\widetilde{T}(S)$ is a stretch flow in $\widetilde{\mathscr{T}}(S)$ directed by $\mu$.

Proof. The transverse measure of the horocyclic foliation coincides with hyperbolic length on geodesic arcs that are perpendicular to the leaves of this foliation, and this transverse measure, under a stretch line, is multiplied by the factor $e^{t}$. Therefore, the shift parameters under a stretch line are multiplied by the same factor.

This means that a stretch line in $\mathscr{T}^{\mu}(S)$ is an open Euclidean ray starting at the origin $O$ and passing through $g$. It can be extended by continuity to a closed ray containing the origin. In particular, all the stretch rays in the shift coordinates associated to $\mu$ converge in the negative direction to the point in $\mathscr{T}^{\mu}(S)$ representing the sphere with three cusps.

It is a natural question to find an expression for the stretch lines on a surface $S_{\mathfrak{g}, \mathfrak{b}, \mathfrak{p}}$ in terms of the Fenchel-Nielsen coordinates. In the next section, we shall see in particular that under a stretch line, the Fenchel-Nielsen twist between adjacent pairs of pants is not constant.

7.2. Earthquakes. Besides the stretch flow, there is a natural flow defined on the unfolded Teichmüller space $\mathscr{T}^{\mu}(S)$, whose effect is to shift any two adjacent ideal triangles relatively to each other in the same direction by the same constant vector. In terms of the shift coordinates, the effect is to add a constant, and it is given by

$$
t \mapsto\left(\delta_{\alpha}, \delta_{\beta}, \delta_{\gamma}\right)+t\left(\delta_{\alpha}, \delta_{\beta}, \delta_{\gamma}\right), \quad t \in \mathbb{R} .
$$

In analogy with Thurston's earthquake flows defined along compactly supported measured laminations, we call this flow an earthquake flow along the ideal triangulation $\mu$. The normalization that we use in this definition makes the origin $O$ of $\mathscr{T}^{\mu}(S)$ (that is, the sphere with three cusps) fixed, and it makes the flow commute with the stretch flow on $\mathscr{T}^{\mu}(S)$. The flowlines are parallel Euclidean lines. 
In general, performing an earthquake along $\mu$ changes the lengths of the boundary components of the surface. In fact, some of these lengths which are nonzero can become 0 as we perform an earthquake. In other words, along an earthquake line, the boundary components can become cusps, and the sense of spiraling of edges of $\mu$ around boundary components can change as the line passes through such a point.

\section{The position of the singular arcs of the horocyclic foliation}

In this section, $S$ is a hyperbolic pair of pants equipped with a measured foliation $F$ transverse to the boundary. A singular arc joining two boundary components of $S$ is an injective arc contained in the leaves of $F$ which joins these boundary components and which passes through a singular point of $F$. In what follows, $F$ will be the horocyclic foliation associated to a hyperbolic structure (with three geodesic boundary components) and to an ideal triangulation $\mu$. Note that since the horocyclic foliation has only three-prong singularities, then for any two boundary components of $S$, there are exactly two singular arcs joining them. (This follows from the classification of measured foliations on pairs of pants, cf. [6]).

Let $g$ be a hyperbolic structure on $S$. Let $A$ and $B$ be two boundary components of $S$, let $k$ and $k^{\prime}$ be the two singular arcs of $F=F_{\mu}(g)$ joining them and let $l$ be the geodesic seam joining these components (see Figure 14).

We shall give a formula for the relative position of one of the singular arcs, say $k$, with respect to $l$. This formula will be useful in studying the relative behaviour of various stretch lines in the Teichmüller space of a surface obtained by gluing pairs of pants along their boundary. It is also used to study the relation of these lines to earthquakes. Recall that stretch lines are naturally expressed using shift coordinates, whereas earthquakes along the curves of the pairs of pants decompositions (that is, Fenchel-Nielsen flows) are naturally expressed using FenchelNielsen coordinates.

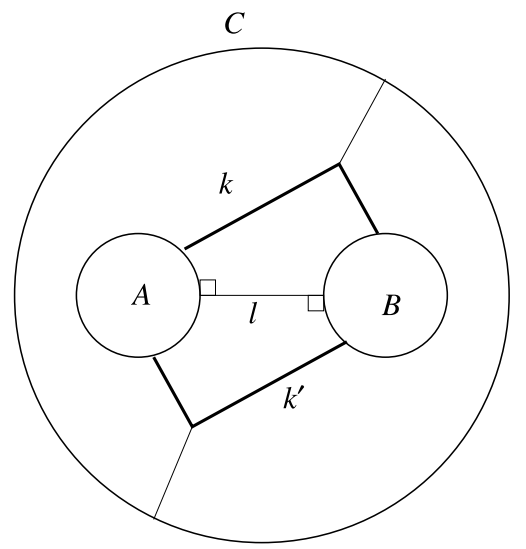

Figure 14. A schematic picture of the singular arcs $k$ and $k^{\prime}$ (in bold lines) and the geodesic arc $l$ joining the two boundary components $A$ and $B$ 


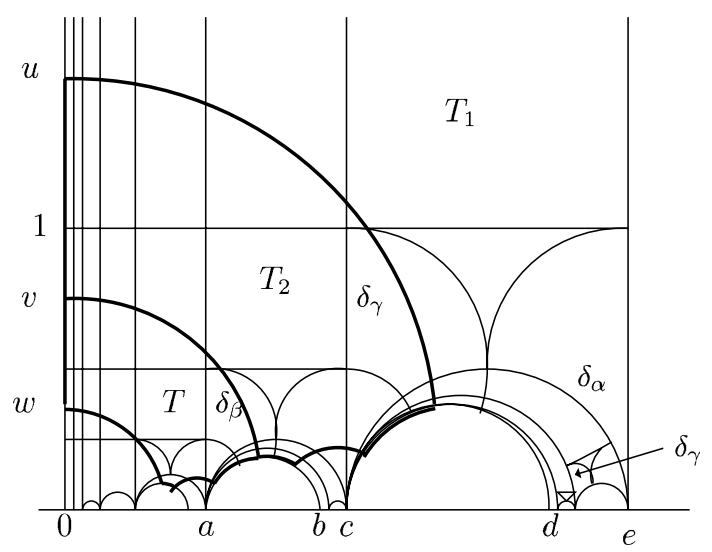

Figure 15. In bold lines, we have represented a fundamental region of the action of the deck transformation group on the universal cover of the pair of pants $S$. This region is the union of lifts of two right-angled hexagons in $S$ obtained by connecting pairwise the three boundary components by geodesic arcs that are perpendicular to these components. The normalization is such that the singular arc $\tilde{k}$ connecting the geodesic lines $\widetilde{A}=(0, \infty)$ and $\widetilde{B}=(c, d)$ intersects the geodesic line $\widetilde{A}$ at height 1 .

We are interested in the ordinate $u$ of the arc $\tilde{l}$ connecting $\widetilde{A}$ and $B$ perpendicularly

In the next section, we shall consider a surface obtained by gluing two pairs of pants along their boundary components. There are at least two useful cooordinates on the Teichmüller space of that surface, the Fenchel-Nielsen coordinates and the shift coordinates. Fenchel-Nielsen coordinates involve distances between endpoints of seams of adjacent pairs of pants, whereas shift coordinates involve distances between endpoints of singular arcs of adjacent pairs of pants. Making the relations between the shift and Fenchel-Nielsen coordinates involves computing distances between endpoints of singular arcs and endpoints of geodesic seams connecting curves in the pairs of pants, and we shall do this below.

The computations are done in the universal covering of the surface $S$. In Figure 15, the universal covering of the hyperbolic pair of pants is represented as a subset of the upper-half plane model of $\mathbb{t}^{2}$. The universal covering is normalized so that the vertical geodesic line $\widetilde{A}$ that joins the points 0 to $\infty$ is a lift of the closed geodesic $A$. The line $\widetilde{A}$ divides $\mathbb{U}^{2}$ into two regions, one of them containing the universal covering of the pair of pants $S$. In Figure 15, this region is the one to the right of $\widetilde{A}$. We choose a lift $\widetilde{B}$ of the boundary component $B$ of $S$ such that the geodesic segment $l$ joining perpendicularly $A$ and $B$ lifts to a segment $\tilde{l}$ joining perpendicularly $\widetilde{A}$ and $\widetilde{B}$. Let $\widetilde{F}$ be the preimage of the foliation $F$ to this universal covering. There are two singular arcs of the horocyclic foliation $\widetilde{F}$ which join the lifts $\widetilde{A}$ and $\widetilde{B}$ of $A$ and $B$. These singular arcs are the two lifts of $k$ and $k^{\prime}$. We let $\tilde{k}$ and $\tilde{k}^{\prime}$ be respectively these arcs. We suppose without loss of generality that the ordinate of the intersection point $\tilde{k} \cap \widetilde{A}$ is greater than the ordinate of the intersection point $\tilde{k}^{\prime} \cap \widetilde{A}$. We compute the signed distance $U$ between the points $\widetilde{A} \cap \tilde{l}$ and $\widetilde{A} \cap \tilde{k}$, with the convention that the orientation on the line $\widetilde{A}$ goes from 0 to $\infty$. Note that this is inverse to the orientation induced from the orientation of the surface. To do these computations, we can fix the ordinate of $\widetilde{A} \cap \tilde{k}$ to be equal to 1 (see Figure 15 in which $u>1$, that is, $U=\log (u)>0$ ). 
We treat in detail the case where the shift coordinates $\delta_{\alpha}, \delta_{\beta}, \delta_{\gamma}$ of the pair of pants are all positive. (This is Case 1 represented in Figure 12). The other cases can be treated by adapting the computations of this special case. We use the notations of Figure 15.

The aim is to compute the value of $u$. Since the three shifts are positive, the spiraling in $S$ around the three boundary geodesics of $S$ is in the inverse sense with respect to the orientation induced by that of the surface.

Figure 15 shows part of the preimage of $\mu$ in the upper half-plane containing the universal covering of the pair of pants. The triangles labeled $T_{1}$ and $T_{2}$ (which have vertical sides) are lifts of the two ideal triangles that are determined by $\mu$ in the surface $S$, and which (for simplicity) have the same name. As already stated, the normalization in the universal covering is such that the lift $\widetilde{A}$ of the boundary component $A$ of $S$ is the vertical geodesic line starting at the origin of the boundary $\mathbb{R}$ of the upper half-plane. We have also drawn lifts of the boundary components $C$ and $B$; these are respectively the geodesics with endpoints $(a, b)$ and $(c, d)$. We start by computing the abscissas $c$ and $d$ in terms of the shifts $\delta_{\alpha}, \delta_{\beta}, \delta_{\gamma}$.

Using the Euclidean theorem of Pythagoras (see Figure 16), we can see that the geodesic segment that minimizes the distance between the vertical line $\widetilde{A}$ and a hyperbolic geodesic which intersects the real line at points whose abscissas are $x$ and $y(0<x<y)$ intersects $\widetilde{A}$ at a point $u$ whose ordinate is $\sqrt{x y}$. Therefore, in our situation (using the notations of Figure 15), we have $u=\sqrt{c d}$.

Using again the notations of Figure 15, we now compute the values of $e, c, a$ and $d$, and we then deduce the value of $u$.

The hyperbolic isometry $z \mapsto f(z)=e^{-l(A)} z$ is a covering transformation that preserves the geodesic $\widetilde{A}$. Under the action of this isometry, the orbits of the vertical sides of the triangles labelled $T_{1}$ and $T_{2}$ in Figure 15 accumulate on the vertical line $\widetilde{A}$. The segment on the $x$-axis with endpoints $e$ and $a$ is a fundamental domain for the action induced by this isometry on the $x$-axis,

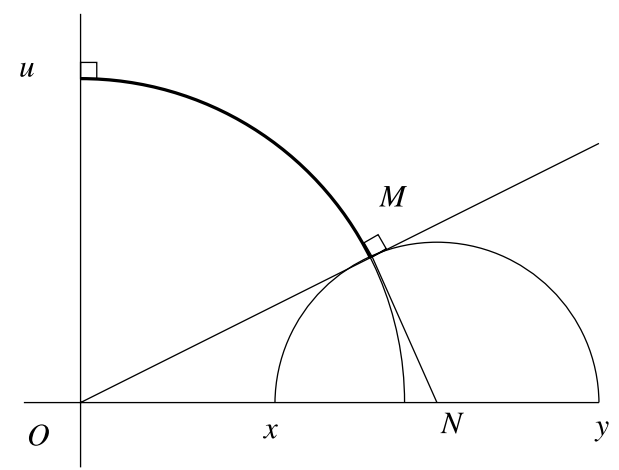

Figure 16. The abscissa of the center $N$ of the Euclidean circle that passes through the points of abscissas $x$ and $y$ equals $(x+y) / 2$, and the radius of this circle is $(y-x) / 2$. By applying the Pythagorean theorem to the Euclidean triangle $O M N$, we obtain $u^{2}=\left(\frac{x+y}{2}\right)^{2}-\left(\frac{y-x}{2}\right)^{2}=\frac{1}{4}(2 y)(2 x)$, which gives $u=O M=\sqrt{x y}$ 
since, by this isometry, the triangle with label $T_{1}$ in Figure 15 is sent to a triangle to the left in that figure. Thus, we have

$$
e=(e-a)+(e-a) e^{-l(A)}+(e-a) e^{-2 l(A)}+\cdots
$$

or, equivalently, using the fact that $l(A)=\delta_{\beta}+\delta_{\gamma}$,

$$
e=(e-a)+\sum_{n=0}^{\infty} e^{-n\left(\delta_{\beta}+\delta_{\gamma}\right)}=\frac{e-a}{1-e^{-\left(\delta_{\beta}+\delta_{\gamma}\right)}}
$$

Using the fact that the length of the largest segment of the horocyclic foliation in the triangle $T_{1}$ whose vertices are $c, e$ and $\infty$ is equal to 1 , we obtain $e-c=1$. Likewise, by examining the triangle $T_{2}$ whose vertices are $a, c$ and $\infty$, we see that $c-a=e^{-\delta_{\gamma}}$.

Now we write $e-a=e-c+c-a=1+c-a=1+e^{-\delta_{\gamma}}$. This gives us

$$
\begin{gathered}
e=\frac{1+e^{-\delta_{\gamma}}}{1-e^{-\left(\delta_{\beta}+\delta_{\gamma}\right)}}, \\
c=e-1=\frac{e^{-\left(\delta_{\beta}+\delta_{\gamma}\right)}+e^{-\delta_{\gamma}}}{1-e^{-\left(\delta_{\beta}+\delta_{\gamma}\right)}}
\end{gathered}
$$

and

$$
a=e^{-\left(\delta_{\beta}+\delta_{\gamma}\right)} e .
$$

To compute the value of $d$, we apply the isometry

$$
z \mapsto g(z)=\frac{-1}{z-c}+\frac{1}{d-c} .
$$

This isometry sends the hyperbolic geodesic joining the points $c$ and $d$ at infinity to the geodesic $\widetilde{A}=(0, \infty)$. Now that the geodesic $g(c d)$ is the vertical line $(0, \infty)$, we can do the computations as we did for the situation described in Figure 15, and we obtain

and

$$
\begin{aligned}
g(e) & =-\frac{1}{e-c}+\frac{1}{d-c} \\
g(\infty) & =\frac{1}{d-c}
\end{aligned}
$$

$$
g(c+i)=-\frac{1}{i}+\frac{1}{d-c}=\frac{1}{d-c}+i .
$$

In particular, the point $g(c+i)$ has ordinate 1 . Therefore, we can compute $g(\infty)$ in the same way we computed $e$ in the preceding situation. We obtain, using now the notations of Figure 17,

$$
g(\infty)=(g(\infty)-x)+(g(\infty)-x) e^{-l(B)}+\cdots=\frac{(g(\infty)-x)}{1-e^{-l(B)}}=\frac{1+e^{-\delta_{\alpha}}}{1-e^{-\left(\delta_{\alpha}+\delta_{\gamma}\right)}} .
$$

Therefore,

$$
d=c+\frac{1-e^{-\left(\delta_{\alpha}+\delta_{\gamma}\right)}}{1+e^{-\delta_{\alpha}}} .
$$




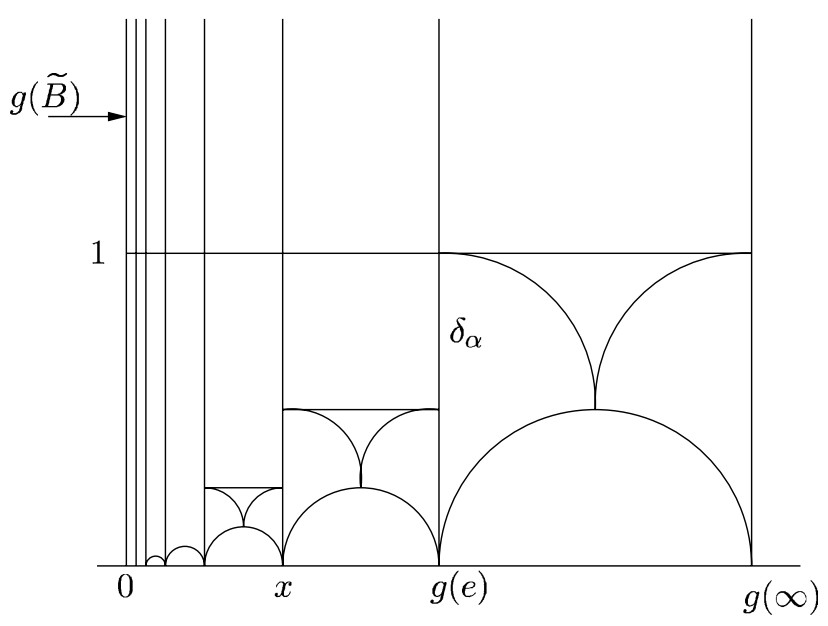

Figure 17

We obtain, after a simplification,

$$
u^{2}=\frac{e^{-\delta_{\gamma}}\left(1+e^{-\delta_{\beta}}\right)\left(1+e^{-\delta_{\gamma}}\right)\left(1+e^{-\left(\delta_{\alpha}+\delta_{\beta}+\delta_{\gamma}\right)}\right)}{\left(1+e^{-\delta_{\alpha}}\right)\left(1-e^{-\left(\delta_{\beta}+\delta_{\gamma}\right)}\right)^{2}} .
$$

From this, we can deduce the value of $U=\log u$.

Note that if $U^{\prime}$ is the signed distance between $l$ and the other singular arc joining $a$ and $b$ (i.e. the arc $k^{\prime}$ ), we have

$$
U^{\prime}=U+\delta_{\gamma} .
$$

Remark 8.1. It is interesting to compare the behaviour of the distance $U$ with that of the length $l(A)$ of the boundary geodesic $A$ as the metric varies on the stretch line $g^{t}$ directed by $\mu$, with $t \rightarrow \infty$. We already know that under a stretch of factor $e^{t}$, the length of $A$ is multiplied by $e^{t}$. Denoting by $r_{t}$ the ratio $U / l(A)$, we have

$$
\lim _{t \rightarrow+\infty} r_{t}=-\frac{\delta_{\gamma}}{2\left(\delta_{\gamma}+\delta_{\beta}\right)} \geq-\frac{1}{2} .
$$

Furthermore, it is easy to see that the function $r_{t}$ is strictly decreasing for $t$ large enough. Thus, along a stretch line, and for $t$ greater than a certain value, the positions of $k$ and $k^{\prime}$ stabilize in the sense that these paths do not wind indefinitely around boundary components.

The situation is totally different along an anti-stretch line. Whereas the length of the boundary component $A$ tends to zero, the distance $U$ goes to infinity. More precisely, one can show that if $l(A)$ denotes the length of the boundary component $A$ at the origin of the anti-stretch line, we have

$$
r_{t} \sim_{t \rightarrow-\infty}-\frac{t e^{-t}}{l(A)}
$$

which tends to infinity as $t \rightarrow-\infty$. 
This behaviour suggests that the horocyclic foliation is not a sufficiently stable tool to study the asymptotic behaviour of an anti-stretch line.

\section{Fenchel-Nielsen coordinates and shift coordinates}

In this section, $S=S_{0,4,0}$ is the sphere with four boundary components equipped with a hyperbolic structure obtained by gluing two hyperbolic pairs of pants $P_{1}$ and $P_{2}$, whose boundary geodesics are denoted respectively by $A_{1}, B_{1}, C_{1}$ and $A_{2}, B_{2}, C_{2}$. We equip each pair of pants with a symmetric ideal triangulation, and the surface $S$ with the union ideal triangulation. We suppose that the geodesics $A_{1}$ and $A_{2}$ have the same lengths, and are identified: $A_{1}=A_{2}=A$. We denote the shift coordinates in $P_{1}$ by $\delta_{\alpha}^{1}, \delta_{\beta}^{1}, \delta_{\gamma}^{1}$, and those in $P_{2}$ by $\delta_{\alpha}^{2}, \delta_{\beta}^{2}, \delta_{\gamma}^{2}$. The gluing condition is $\left|\delta_{\beta}^{1}+\delta_{\gamma}^{1}\right|=$ $\left|\delta_{\beta}^{2}+\delta_{\gamma}^{2}\right|$. The union of the two ideal triangulations of $P_{1}$ and $P_{2}$ defines a geodesic lamination on $S$. This lamination is chosen such that all the spirals of $P_{1}$ and $P_{2}$ are positive and all the spirals around $A_{1}=A_{2}$ that come from the two adjacent pairs of pants are in the same direction (that is, the lamination is chain recurrent).

There are at least two ways of specifying the gluing between $P_{1}$ and $P_{2}$, that is, of describing the twist along $A$ between these two pairs of pants. First, we have the Fenchel-Nielsen twist $\tau_{A}$, which measures the signed distance on $A$ between the feet of the geodesic seams of $P_{1}$ and $P_{2}$ (after the choice of a pair of boundary components in each pair of pant) and then, we have the shift twist $\delta_{A}$, which measures the signed distance on $A$ between the feet of the singular arcs of the horocyclic foliations (again, after making choices).

In this section, we shall give a formula that relates these two twists. Recall that the shift twist $\delta_{A}$ along $A$ is natural for describing stretches.

First, one has to choose a geodesic arc in each pair of pants: we shall choose the seam joining $A$ and $B_{1}$ on one side of $A$ and the seam joining $A$ and $B_{2}$ on the other side. Likewise, we choose one singular arc on each side of $A$, joining the same boundary components of the pair of pants as the geodesic arcs that we choose.

Moreover, we choose an orientation on the closed geodesic $A$.

We note that these choices do not suffice to determine the Fenchel-Nielsen and the shift parameters. One also needs to choose an origin for each of these shifts. The choices become natural if we lift the situation to the universal covering of the surface. First consider a lift $\widetilde{A}$ of $A$. This lift separates the universal covering into two half-planes bounded by $\widetilde{A}$. In each of these half-planes, there is a whole family of lifts of seams with one endpoint on $\widetilde{A}$, all of these lifts being congruent under the action of the cyclic subgroup of the deck transformations group that preserves $\widetilde{A}$. The choices that we have to make for the definition of the Fenchel-Nielsen coordinates amount to choosing an orientation on $\widetilde{A}$ and two representatives, one in each half-plane, of the two families of these lifts of the seams. The choices made for one lift propagate under the action of the deck-transformations group.

Of course, the determination of the shift twist parameters is based on an analogous battery of choices.

We want to compare the two twist parameters, and there is a convenient way to do so. Once we have made choices to define one type of parameters, there is a convenient way for making the choices defining the other type of parameters. 

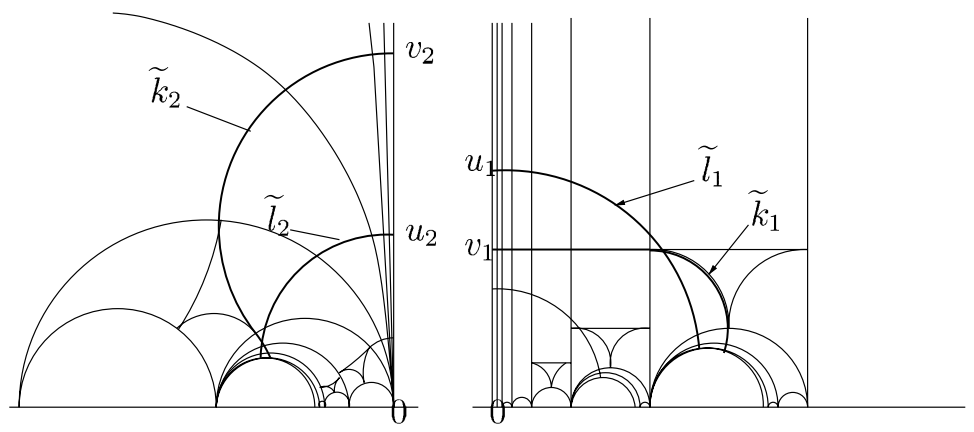

Figure 18. To pass from the left hand figure to the right hand figure, we use the isometry $z \mapsto-1 / z$. Thus, $a^{\prime}=-1 / a, b^{\prime}=-1 / b$ and so on. Therefore, $1 / u^{\prime}=\sqrt{\left(-1 / c^{\prime}\right)\left(-1 / d^{\prime}\right)}$, i.e., $u^{\prime}=\sqrt{c^{\prime} d^{\prime}}$

Indeed, each type of segment (seam or singular arc) used in the definition of the corresponding type of parameters joins two boundary components of the pair of pants containing this segment. Each lift of such a segment joins lifts of those boundary components. If one chooses two particular lifts of the boundary components to define the parameters, then it is natural to choose a segment of the other type joining the same lifts. Thus, in what follows, we will apply the following procedure: we first make choices for the definition of the Fenchel-Nielsen twist parameters, and then make choices for the definition of the shift twist parameters accordingly. This will allow us to use the computations made in the previous section.

We now consider the lift of this situation to the universal cover $\widetilde{S}$ of $S$. We choose an identification between $\widetilde{S}$ and a subset of the upper half-plane $\mathbb{U}^{2}$ in such a way that a lift $\widetilde{A}$ of $A$ is the geodesic whose endpoints are at $0, \infty$. We choose a lift $\tilde{l}_{i}, i=1,2$, of a seam in each pair of pants in order to compute the FenchelNielsen twist. The orientation of $A$ corresponds to the orientation of $\widetilde{A}$ pointing from 0 to $\infty$. In Figure 18, the subset of the universal cover of $P_{1}$ containing $\widetilde{A}$ is situated to the right of $\widetilde{A}$, and that of $P_{2}$ to the left. The picture on the right is put in the same position as in Figure 15, that is, the lifts of the edges spiraling around $A$ (which are geodesics accumulating on $\widetilde{A}$ ) have $\infty$ as an endpoint. We refer to the position pictured in Figure 15 as the "standard position". The picture on the left is not in the standard position, but it can be made so by using the map $z \mapsto-1 / z$.

We make the choices of the singular segments according to the discussion above and to Figure 18, namely, we choose among the two possible singular segments joining the chosen lifts of boundary components of pairs of pants. In $P_{1}$, we take the singular segment with the highest point of intersection with $\widetilde{A}$, and we make the same choice in $P_{2}$ after having put it in the standard position. We call $\tilde{k}_{i}, i=1,2$, the singular segments chosen $i_{\tilde{l}}$ this way. and we let $u_{i}, v_{i}$ denote the ordinates of the points of intersection of $\tilde{l}_{i}$ and respectively $\tilde{k}_{i}$ with $\widetilde{A}$. Then, we have (see Figure 19 where the geodesic $\widetilde{A}$ is oriented from bottom to top):

- $\tau_{A}=\log \left(\frac{u_{2}}{u_{1}}\right)$,

- $\delta_{A}=\log \left(\frac{v_{2}}{v_{1}}\right)$,

- $U_{1}=\log \left(\frac{v_{1}}{u_{1}}\right)$,

- $U_{2}=\log \left(\frac{v_{2}}{u_{2}}\right)$, 


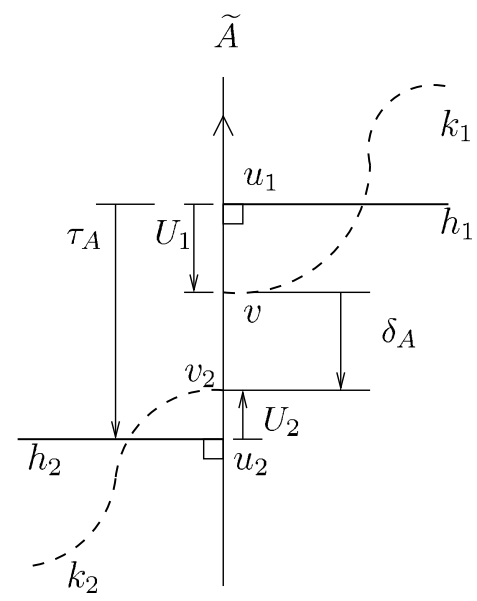

Figure 19. Gluing two copies of the the universal coverings of the two pairs of pants along $\widetilde{A}$. The dotted lines represent lifts of the singular arcs and the perpendicular arcs represent lifts of the seams. The directions of the arrow represent positive values of the various quantities. Using the orientation of the geodesic $\tilde{A}$ and the orientation of the surface, the preferred side of $\tilde{A}$ lies here on the left-hand side.

In this figure, the arrangement of the curves $\tilde{h}_{i}, \tilde{k}_{i}, i=1,2$, gives $U_{1}<0, U_{2}>0, \tau_{A}<0, \delta_{A}<0$

where $U_{i}$ denotes the signed distance from the foot of the seam $\tilde{l}_{i}$ on $\widetilde{A}$ to the foot of the singular segment $\tilde{k}_{i}$ on $\widetilde{A}$. The sign is determined using the orientation on $\tilde{A}$. This orientation allows one to distinguish a preferred side to that curve, namely, the side which induces an orientation on $\tilde{A}$ compatible with the chosen one. The sign of the shift is then obtained by considering in which direction the preferred side is shifted with respect to the orientation of $\tilde{A}$. Therefore, we have

$$
\tau_{A}=\delta_{A}+U_{1}-U_{2} .
$$

\section{An example on the surface of genus 2}

Let $P$ be a hyperbolic pair of pants with geodesic boundary components $A, B, C$ equipped with a complete geodesic lamination $\mu$ of symmetric type (that is, no leaf joins a puncture to itself). As before, $\delta_{\alpha}, \delta_{\beta}, \delta_{\gamma}$ denote the shift parameters associated to $\mu$. Consider a hyperbolic structure on $P$ satisfying the following conditions:

(1) the shifts are all positive, i.e., $\delta_{\alpha}>0, \delta_{\beta}>0, \delta_{\gamma}>0$;

(2) the lengths of all the boundary components are equal to some $L>0$, i.e., $\delta_{\alpha}+\delta_{\beta}=\delta_{\alpha}+\delta_{\gamma}=\delta_{\beta}+\delta_{\gamma}=L$.

Note that we then have $\delta_{\alpha}=\delta_{\beta}=\delta_{\gamma}=L / 2$.

We take two copies $P_{1}$ and $P_{2}$ of $P$, and we call respectively $\mu_{1}$ and $\mu_{2}$ the laminations in these two pairs of pants corresponding to $\mu$. We glue pairwise the boundary components of $P_{1}$ with the boundary components of $P_{2}$, respecting the labels of these components. We denote by $S=S_{2}$ the resulting surface. It is a closed surface of genus 2 . We can naturally talk about the geodesics $A, B, C$ in $S$. Note that there is one degree of freedom for each identification between boundary 
components of $P_{1} \cup P_{2}$ and that for any choice of identifications between these components, the union of the complete laminations $\mu_{1}$ and $\mu_{2}$ is a complete geodesic lamination on $S$, which we also denote by $\mu$, and which we shall suppose to be chain-recurrent (i.e. all leaves of $\mu$ that spiral along the same closed geodesic $A, B$ or $C$ spiral in the same direction). We choose the identifications between the boundary components of $P_{1}$ and $P_{2}$ in such a way that the seams have the same endpoints, thus forming three disjoint simple closed geodesics $A^{\prime}, B^{\prime}, C^{\prime}$, each of which have at most one intersection point with the closed geodesics $A, B, C$ $\left(A^{\prime} \cap A=\emptyset, B^{\prime} \cap B=\emptyset, C^{\prime} \cap C=\emptyset\right)$. Finally, let us denote by $h$ the resulting hyperbolic structure on $S$. (Recall that $h$ is only defined up to isometry).

The hyperbolic surface $h$ has two order-two symmetries. The first one, $\sigma$, fixes the geodesics $A, B, C$ and exchanges the (images in $S$ of the) pairs of pants $P_{1}$ and $P_{2}$. The second one, $\sigma^{\prime}$, fixes $A^{\prime}, B^{\prime}, C^{\prime}$ and its restriction to each pair of pants $P_{1}$, $P_{2}$ is the canonical involution fixing the seams.

Let $\bar{\mu}$ be the image of $\mu$ by the symmetry $\sigma^{\prime}$ of $S$. The geodesic lamination $\bar{\mu}$ is also obtained from $\mu$ by reversing the sense of spiraling around the three closed geodesics $A, B, C$. Note that if $\bar{\delta}_{\alpha}, \bar{\delta}_{\beta}, \bar{\delta}_{\gamma}$ denote the shift parameters associated to $\bar{\mu}$, then we can easily see that we have

$$
\bar{\delta}_{\alpha}=-\delta_{\alpha}, \quad \bar{\delta}_{\beta}=-\delta_{\beta}, \quad \bar{\delta}_{\gamma}=-\delta_{\gamma} .
$$

We now consider the two stretch lines $\ell: t \mapsto h_{t}$ and $\bar{\ell}: t \mapsto \bar{h}_{t}$ starting at $h=h_{0}=\bar{h}_{0}$ and directed by $\mu$ and $\bar{\mu}$ respectively. We shall study explicitely the Fenchel-Nielsen coordinates of the points $h_{t}$ and $\bar{h}_{t}$ on these lines, associated to the pair of pants decomposition $A, B, C$. Note that since $l_{i}\left(h_{t}\right)=l_{i}\left(\bar{h}_{t}\right)$, for $i=A, B, C$, we can always pass from $h_{t}$ to $\bar{h}_{t}$ by a composition of Fenchel-Nielsen twists along the curves $A, B$ and $C$, but it is a priori conceivable that $h_{t}=\bar{h}_{t}$ for some (or even for all) $t$, in addition to $t=0$. For instance, it is conceivable that a stretch map does not change the Fenchel-Nielsen parameters between adjacent pairs of pants. In fact, we shall show that this is not the case. We shall see that the twist parameters along $A, B$ and $C$ are always different for points on $\ell$ and on $\bar{\ell}$ except at the origin, thus proving the following

Proposition 10.1. The geodesic lines $\ell$ and $\bar{\ell}$ have a unique point of intersection, which is the point $h$.

Proof. Consider the Fenchel-Nielsen coordinates $\left(l_{A}, \tau_{A}, \ldots, l_{C}, \tau_{C}\right)$ associated to the three closed curves $A, B, C$, such that $\tau_{A}(h)=\tau_{B}(h)=\tau_{C}(h)=0$.

By Proposition 7.1, we have, for each $t \geqslant 0$,

$$
l_{i}\left(h_{t}\right)=l_{i}\left(\bar{h}_{t}\right)=e^{t} L, \text { with } i=A, B, C .
$$

We shall show that the Fenchel-Nielsen twist parameters $\tau_{i}\left(h_{t}\right)$ and $\tau_{i}\left(\bar{h}_{t}\right)$, for any $i=A, B, C$, are different for all $t>0$. For this, we use the formulae that give these twist parameters in terms of the shift coordinates. The situation being the same for the three geodesics $A, B$ and $C$, it suffices to consider one of them, say, $\tau_{A}$.

To compute the shift parameters $\delta_{A}\left(h_{t}\right)$ and $\delta_{A}\left(\bar{h}_{t}\right)$ along $A$, we must choose a singular arc in each pair of pants $P_{1}$ and $P_{2}$ joining $A$ to another boundary component of this pair of pants with respect to the two horocyclic foliations $F_{\mu}(h)$ and 
$F_{\bar{\mu}}(h)$. We shall make such a choice for the structure $h$, and we shall then keep the same choice as $t$ varies. There is a natural choice for the singular arcs with respect to $F_{\bar{\mu}}(h)$ once singular arcs with respect to $F_{\mu}(h)$ have been chosen: to a singular arc in one pair of pants with respect to $F_{\mu}(h)$, we associate the singular arc with respect to $F_{\bar{\mu}}(h)$ of the same pair of pants that joins the same curves in the
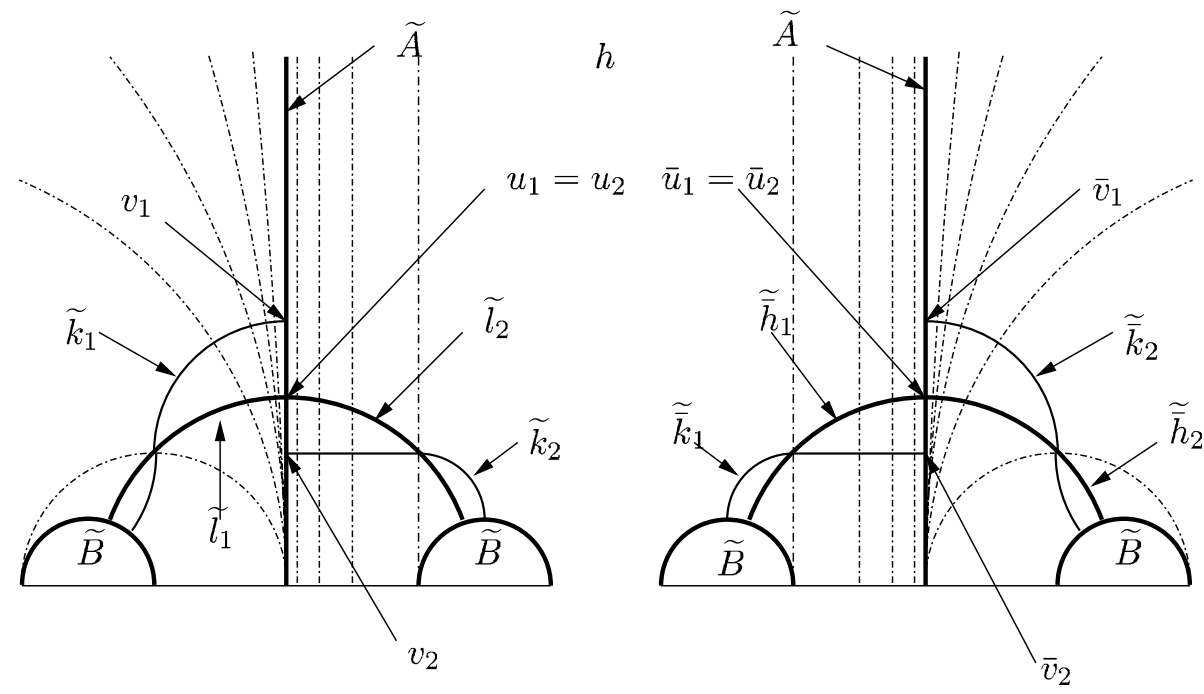

Figure 20. Both pictures represent, in the metric universal cover of $h$, a neighborhood of a lift $\widetilde{A}$ of $A$. The left-hand side picture shows a part of the preimage of $\mu$ (in dashed lines) and the right-hand side picture shows the same neighborhood but with a part of the preimage of $\bar{\mu}$ (also in dashed lines). The (lifts of the) seams $\tilde{h}_{i}, \tilde{h}_{i}^{\prime}, i=1,2$, and the singular arcs $\tilde{k}_{i}, \tilde{k}_{i}^{\prime}, i=1,2$, chosen to evaluate the various shift and twist parameters are indicated, as well as the ordinates of their points of intersection with $\widetilde{A}$ (denoted by $u_{i}, v_{i}, i=1,2$, and $u_{i}^{\prime}, v_{i}^{\prime}, i=1,2$, ). By symmetry, one has $U_{1}=-U_{2}$ and, similarly, $\bar{U}_{1}=-\bar{U}_{2}$. Furthermore, by symmetry again, one has $U_{1}=\bar{U}_{2}$
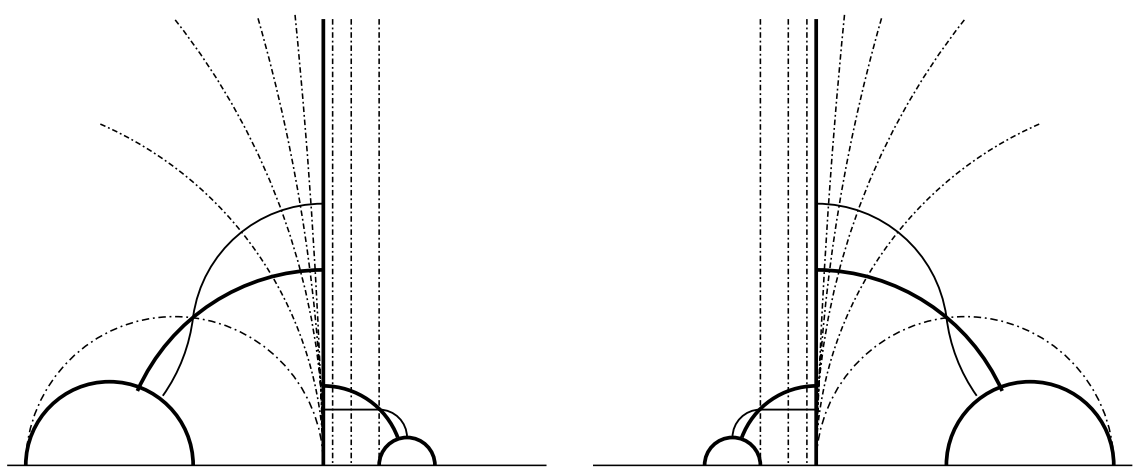

Figure 21. The left-hand side picture represents a neighborhood of a lift of $A$ in the metric universal cover of $h_{t}$ and the right-hand side picture represents a neighborhood of a lift of $A$ in the metric universal cover of $\bar{h}_{t}$. The notations of Fig. 20 carry out readily to these pictures. One observes that there is a symmetry between the two pictures, namely, a reflection through $\widetilde{A}$ 
collection $\{A, B, C\}$ and whose ends on each curve are exchanged by the involution $\sigma^{\prime}$ of $h$.

Note that this involution sends $F_{h}(\mu)$ to $F_{h}(\bar{\mu})$.

By symmetry, we have

(1) $\delta_{A}(h)=-2 U=\frac{L}{2}-\log \left(1+\frac{1}{4 \sinh ^{2}(L / 4)}\right)$ (see Figure 20);

(2) for every $t \geqslant 0, \tau_{A}\left(h_{t}\right)=-\tau_{A}\left(\bar{h}_{t}\right)$ (see Figure 21 ).

Using the fact that the shift twists always have opposite signs, it suffices, in order to prove the proposition, to see that for each $t>0$, we have $\tau_{A}\left(h_{t}\right) \neq 0$. Using the notation of Figure 21, we have $\delta_{A}\left(h_{t}\right)=\tau_{A}\left(h_{t}\right)-2 U(t)$, where $U(t)$ denotes the distance between the foot of the chosen seam and the foot of the chosen singular arc. From the definition of a stretch line, we have $\delta_{A}\left(h_{t}\right)=e^{t} \delta_{A}(h)$. Therefore,

$$
\tau_{A}\left(h_{t}\right)=e^{t} \delta_{A}(h)+2 U(t),
$$

where $2 U(t)=-\frac{e^{t} L}{2}+\log \left(1+\frac{1}{4 \sinh ^{2}\left(e^{t} L / 4\right)}\right)$ and $U(0)=U=\frac{-\delta_{A}(h)}{2}$.

In particular, for all $i=A, B, C$ and for all $t \geqslant 0$, we have

$$
\tau_{i}\left(h_{t}\right)=\log \left(\frac{1+\frac{1}{4 \sinh \left(e^{t} L / 4\right)}}{\left(1+\frac{1}{4 \sinh (L / 4)}\right)^{e^{t}}}\right) .
$$

We can check that this function is strictly decreasing, positive for $t<0$ and negative for $t>0$ (see the graph in Figure 22).

Indeed, we have

$$
\begin{aligned}
& \forall t<0,1+\frac{1}{4 \sinh \left(e^{t} L / 4\right)}>1+\frac{1}{4 \sinh (L / 4)}>\left(1+\frac{1}{4 \sinh (L / 4)}\right)^{e^{t},} \\
& \forall t>0,1+\frac{1}{4 \sinh \left(e^{t} L / 4\right)}<1+\frac{1}{4 \sinh (L / 4)}<\left(1+\frac{1}{4 \sinh (L / 4)}\right)^{e^{t},}
\end{aligned}
$$

which determines the sign of the function.

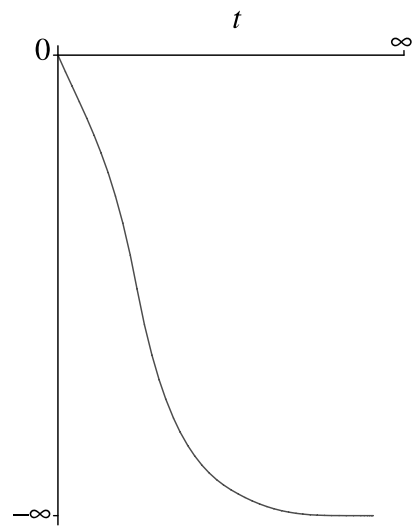

Figure 22. The graph of the function $\tau_{i}\left(h_{t}\right)$ used in the proof of Proposition 10.1 
For the strict decreaseness, $\frac{d}{d t} \tau_{i}\left(h_{t}\right)$ has the same sign as

$$
\frac{d}{d t}\left(\frac{1+\frac{1}{4 \sinh \left(e^{t} L / 4\right)}}{\left(1+\frac{1}{4 \sinh (L / 4)}\right)^{e^{t}}}\right)
$$

which has the sign of

$$
\begin{aligned}
& \left.\left(\frac{d}{d t}\left(1+\frac{1}{4 \sinh \left(e^{t} L / 4\right)}\right)\right)\left(1+\frac{1}{4 \sinh (L / 4)}\right)^{e^{t}}\right) \\
& \left.\quad-\left(1+\frac{1}{4 \sinh \left(e^{t} L / 4\right)}\right)\left(\frac{d}{d t}\left(1+\frac{1}{4 \sinh (L / 4)}\right)^{e^{t}}\right)\right) .
\end{aligned}
$$

We have

$$
\frac{d}{d t}\left(1+\frac{1}{4 \sinh \left(e^{t} L / 4\right)}\right)=-\frac{L e^{t} \cosh \left(e^{t} L / 4\right)}{16 \sinh ^{2}\left(e^{t} L / 4\right)}<0
$$

and

$$
\left(\frac{d}{d t}\left(1+\frac{1}{4 \sinh (L / 4)}\right)^{e^{t}}\right)>0
$$

This concludes the proof of the proposition.

Remarks 10.2. (1) The Fenchel-Nielsen twist parameters are not linear in terms of the shift parameters. More precisely, we have $l_{A}=\delta_{\beta}+\delta_{\gamma}, l_{B}=\delta_{\gamma}+\delta_{\alpha}$ and $l_{C}=\delta_{\alpha}+\delta_{\beta}$,

$$
\tau_{A}=\delta_{A}-\left(\delta_{\beta}+\delta_{\gamma}\right)+2 \log \left(1+\frac{1}{4 \sinh ^{2}\left(\left(\delta_{\beta}+\delta_{\gamma}\right) / 4\right)}\right) .
$$

(2) The formula for $\tau_{i}\left(h_{t}\right)$ given in the proof of Proposition 10.1 indicates the following:

- $\lim _{t \rightarrow+\infty} \tau_{i}\left(h_{t}\right)=-\infty$ and

- $\tau_{i}\left(h_{t}\right) / l_{i}\left(h_{t}\right)$ is bounded (and is convergent at infinity).

- $\lim _{t \rightarrow-\infty} \tau_{i}\left(h_{t}\right)=+\infty$ and

- $\tau_{i}\left(h_{t}\right) / l_{i}\left(h_{t}\right) \sim-t e^{-t}$ explodes to $\infty$ as $t$ tends to $-\infty$.

\section{References}

[1] Baseilhac S, Benedetti R (2008) Teichmüller space and cylinders in quantum hyperbolic field theories. In: Papadopoulos A (ed) Handbook of Teichmüller theory, Vol 2. European Mathematical Society, to appear

[2] Bonahon F, Liu X (2008) Representations of the quantum Teichmuller space and invariants of surface diffeomorphisms. Preprint

[3] Chekhov L, Fock VV (1999) Quantum Teichmüller Space. Theor Math Phys 120: 1245-1259

[4] Chekhov L, Penner RC (2007) On quantizing Teichmüller and Thurston theories. In: Papadopoulos A (ed) Handbook of Teichmüller theory, Vol 1, pp 579-645. Zürich: European Math Soc

[5] Fock VV, Goncharov AB (2007) Dual Teichmüller and lamination spaces. In: Papadopoulos A (ed) Handbook of Teichmüller theory, Vol 1, pp 647-684. European Mathematical Society 
346 A. Papadopoulos and G. Théret: Shift coordinates, stretch lines and polyhedral structures

[6] Fathi A, Laudenbach F, Poénaru (1979) Travaux de Thurston sur les surfaces. Astérisque 66-67

[7] Fenchel W, Nielsen J (2003) Discontinuous groups of isometries in the hyperbolic plane. In: Schmidt AL (ed) Berlin: de Gruyter

[8] Luo F (2007) On Teichmüller space of surface with boundary. Duke Mathematical J 139: 463-482

[9] Papadopoulos A (1991) On Thurston's boundary of Teichmüller space and the extension of earthquakes. Topology and its Applications 41: 147-177

[10] Penner RC (1993) Universal constructions in Teichmüller theory. Adv Math 98: 143-215

[11] Thurston WP (1985) Minimal Stretch maps between hyperbolic surfaces. Preprint available at http://arxiv.org/abs/math.GT/980103

[12] Thurston WP (1997) Three-Dimensional Geometry and Topology, Vol 1. Princeton, Univ Press

Authors' addresses: A. Papadopoulos, Institut de Recherche Mathématique Avancée, Université Louis Pasteur and CNRS, 7 rue René Descartes, 67084 Strasbourg Cedex, France and Max-Planck-Institut für Mathematik, Vivatsgasse 7, 53111 Bonn, Germany, E-mail: papadopoulos@math.u-strasbg.fr; G. Théret, Institut de Recherche Mathématique Avancée, Université Louis Pasteur and CNRS, 7 rue René Descartes, 67084 Strasbourg Cedex, France and Department of Mathematical Sciences, University of Aarhus, Ny Munkegade, Building 1530, DK-8000 Aarhus C, Denmark, E-mail: theret@imf.au.dk 